\title{
Cardinal Contests
}

\author{
ARPITA GHOSH, Cornell University, USA \\ PATRICK HUMMEL, Google Inc., USA
}

\begin{abstract}
We model and analyze cardinal contests, where a principal running a rank-order tournament has access to an absolute measure of the quality of agents' submissions in addition to their relative rankings. We show that a mechanism that compares each agent's output quality against a threshold to decide whether to award her the prize corresponding to her rank is optimal amongst the set of all mixed cardinal-ordinal mechanisms where the $j$ th-ranked submission receives a fraction of the $j$ th prize that is a non-decreasing function of the submission's quality. Furthermore, the optimal threshold mechanism uses exactly the same threshold for each rank.
\end{abstract}

\section{CCS Concepts: • Applied computing $\rightarrow$ Economics;}

Additional Key Words and Phrases: Contests, optimal contest design, crowdsourcing, game theory

\section{ACM Reference format:}

Arpita Ghosh and Patrick Hummel. 2018. Cardinal Contests. ACM Trans. Econ. Comput. 6, 2, Article 7 (July 2018), 32 pages.

https://doi.org/10.1145/3232862

\section{INTRODUCTION}

Contests have a long history as a means for procuring innovations, with government-sponsored contests for research and development dating back to at least $1714 .{ }^{1}$ Contests provide an effective incentive structure for eliciting effort in settings where the quality of an agent's output as well as her effort are unverifiable or difficult to measure, making conventional contracts based on input- or output-dependent reward infeasible-either by virtue of being too costly for the sponsor to implement, or because they cannot be credibly enforced due to unverifiability of output [9, 49]. In several such situations, an ordinal comparison-identifying a relative ranking of agents' submissionsmight nonetheless be feasible, allowing the principal to commit to an enforceable contract that awards rank-based prizes to some subset of entrants in a contest. This has led to a large literature on the optimal design of rank-order mechanisms for effort elicitation; see Section 1.1.

In contrast with more traditional settings, however, an increasing number of online contests procure innovations whose quality is evaluated and ranked via verifiable cardinal measurements. For instance, the well-known Netflix contest, designed to procure improved algorithms for movie

\footnotetext{
${ }^{1}$ The British parliament ran a contest with a 20,000 pound prize for a method for determining longitude-at-sea to within half a degree.

Authors' addresses: A. Ghosh, Cornell University, 206 Gates Hall, Ithaca NY 14853 USA; email: arpitaghosh@cornell.edu; P. Hummel, Google Inc., 1600 Amphitheatre Parkway, Mountain View, CA 94043 USA; email: phummel@google.com.

Permission to make digital or hard copies of all or part of this work for personal or classroom use is granted without fee provided that copies are not made or distributed for profit or commercial advantage and that copies bear this notice and the full citation on the first page. Copyrights for components of this work owned by others than the author(s) must be honored. Abstracting with credit is permitted. To copy otherwise, or republish, to post on servers or to redistribute to lists, requires prior specific permission and/or a fee. Request permissions from permissions@acm.org.

2018 Copyright is held by the owner/author(s). Publication rights licensed to ACM.

ACM 2167-8375/2018/07-ART7 \$15.00

https://doi.org/10.1145/3232862
} 
recommendations, evaluated entries according to how well they predicted user preferences on a test subset of its user database, with the algorithm that obtained the highest score being declared the winner. In a different context, contests for designing mobile apps for health or education might compute scores for submissions based on their performance on metrics of efficacy in randomized trials, ${ }^{2}$ and rank entries based on these scores. A third example is the contest platform Kaggle, which hosts contests where the innovation being procured is a data-mining algorithm: again, submissions are typically evaluated on a test dataset provided by the requesters and ranked according to the score they obtain. A number of other such contests abound. In summary, there is an increasingly large family of contests where submissions can be assigned a meaningful numerical quality score that reflects their value to the principal.

Suppose a principal running a contest has access to such cardinal measurements of the qualities of agents' submissions, in addition to the ordinal ranking of their outputs. What effect does making prizes contingent on absolute performance, in addition to relative ranking, have on contestants' incentives for effort, and what is the optimal way to incorporate such cardinal measurements of output into an ordinal rank-order contest?

Our contributions. We model and analyze cardinal contests, where a principal, who wants to maximize some increasing function of the quality of received submissions, can evaluate the quality of each submission via a real-valued score in addition to observing the rank-ordering of contestants' outputs. We ask whether and how such a principal can improve his utility by incorporating such cardinal information to determine agents' rewards.

Specifically, consider a rank-order tournament with prizes $\left(A_{1}, A_{2}, \ldots, A_{n}\right)$ for ranks $1, \ldots, n$, in a model where contestants are strategic and have a cost to effort. Can the principal modify the rank-order mechanism $\mathcal{M}\left(A_{1}, A_{2}, \ldots, A_{n}\right)$, using participants' quality scores $q_{j}$ in addition to their ranks $j$ to decide how much of the prize $A_{j}$ to actually award, to improve incentives for effort? And if so, how should the function $g_{j}\left(q_{j}\right)$ that determines the fraction of the prize $A_{j}$ to be awarded to the $j$ th-ranked contestant be chosen to optimally incentivize the agents?

Our main result is that a very simple threshold mechanism-a mechanism that awards the prize for a rank if and only if the output of the agent at that rank exceeds a certain threshold-is optimal amongst all mechanisms induced by any non-decreasing functions $g_{j}(\cdot)$ : We show that for any given rank-order mechanism $\mathcal{M}\left(A_{1}, A_{2}, \ldots, A_{n}\right)$, the functions $g_{j}\left(q_{j}\right)$ that incentivize the highest effort are precisely step functions that increase from 0 to 1 at a threshold score (Theorem 3.1). Additionally, if the noise density that stochastically maps an agent's effort into her output quality is single-peaked at 0 , we find that the optimal threshold mechanism also uses exactly the same threshold for each rank, meaning the optimal threshold mechanism would effectively remove all agents whose quality falls below a certain threshold, and rank and award prizes to the remaining contestants as per the original mechanism. Interestingly, real-world contests where prizes are made contingent on cardinal information measuring absolute performance seem to commonly use such threshold-based prize structures. ${ }^{3}$

After deriving comparative statics for the optimal threshold and equilibrium effort in such mechanisms in Section 4.1, we ask to what extent does incorporating cardinal information (via an optimal threshold mechanism) improve equilibrium effort relative to using only ordinal information? We address this question via numerical simulations as well as theory, and find that the extent to which cardinal information incentivizes higher effort depends on the contest parameters: the most

\footnotetext{
${ }^{2}$ See, for example, http://www.robinhood.org/prize.

${ }^{3}$ For example, one of the largest contests hosted on Kaggle awarded prizes to the top three entries, provided their scores were above a minimum baseline.
} 
substantial improvements are achieved in contests with a smaller number of participants, contests with participants whose cost functions are "not too convex" (in a sense made precise in Section 5), and contests that award a larger rather than smaller number of prizes. We conclude by addressing questions related to whether one would want to modify the prize structure in a rank-order tournament if one wishes to also incorporate cardinal information in Section 6 and by analyzing questions on endogenous entry in Section 7.

We note that we ask the question of how to optimally modify a given rank-order mechanism which awards a prize of $A_{j}$ to the $j$ th-ranked contestant, in the sense that we ask how to optimally use the participants' quality scores $q_{j}$ to decide how much of the $j$ th prize to award to the $j$ th-ranked contestant. This contrasts with the question of choosing the overall optimal reward structure $\left(A_{1}\left(q_{1}, \ldots, q_{n}\right), \ldots, A_{n}\left(q_{1}, \ldots, q_{n}\right)\right)$ that incorporates all available cardinal information in determining the reward for each rank. There are a number of reasons we address the question we focus on, the foremost of which is practical: a principal announcing a contest might choose, or be committed to, a certain rank-based prize structure for reasons such as sponsorship constraints, publicity, simplicity, cost of precise evaluations of a full rank-order due to scale, and so on. However, the principal might still want to, and more easily be able to, incorporate an entry's absolute quality in determining whether, and how much of the rank-based prize to actually award (for instance, he may wish to award no prize if the highest-ranked submission performs worse than the current state-of-the-art). See Sections 2 and 8 for further discussion.

\subsection{Related Work}

There is a large body of work on contest design in the economics literature. In addition to general work on the theory of contests [11, 20, 23, 28, 30-33, 38, 41, 43-48], there has also been a variety of work motivated by specific applications. References [19], [26], [27], and [35] address the design of rank-order tournaments for the purpose of incentivizing employees to work hard. References [9] and [49] study contest design in the context of research tournaments. And there is a growing literature motivated by online crowdsourcing contests $[2,7,8,12,18]$. There is also an extensive empirical and experimental literature analyzing observed strategic behavior by real subjects in contests in a variety of settings $[1,4-6,10,14,15,21,29,34,39,40,42]$.

This work addresses a variety of questions on the economics of contests such as comparing tournaments to schemes for incentivizing effort that are based solely on an individual's personal performance [20, 27, 35, 41], relationships between contests and all-pay auctions [8, 9, 12], taxing entry to improve the quality of contributions [18, 49], dynamic contests in which agents dynamically decide how much effort to exert when they get information over time about how they are doing in the contest compared to their competitors [16, 17, 24, 51], and incentives for agents to work hard in teams $[22,36]$. The most relevant subset of this literature to our article is that related to optimal contest design [2, 11, 19, 23, 25, 28, 30-33, 48], which asks how to best choose the rank-based rewards under various models of effort and constraints on the rewards.

The key difference between this literature and our work is that this literature almost exclusively studies contests that are structured as rank-order mechanisms, where the announced prizes depend only on the rank of an agent's output relative to that of her competitors, whereas we consider mixed cardinal-ordinal mechanisms of the form described in Section 2. Specifically, rather than ask which ordinal rewards $\left(A_{1}, A_{2}, \ldots, A_{n}\right)$ incentivize optimal outcomes, we ask how cardinal information about an agent's output can be optimally incorporated into a given ordinal reward mechanism $\mathcal{M}\left(A_{1}, A_{2}, \ldots, A_{n}\right)$ to incentivize the highest effort, a question that has not been addressed previously in this literature. The only relevant exception, to the best of our knowledge, is Reference [8], which does address the question of optimal contest design using both cardinal and ordinal information, albeit in a completely different model of output and for risk-neutral agents 
only. Interestingly, although the models are completely different, the optimal mechanism in that model also turns out to use cardinal information via a threshold.

\section{MODEL}

We consider a setting where a pool of agents competing in a contest strategically choose their effort levels, which stochastically determine their submission qualities, and each agent receives a prize based on the relative rank of her output as well as possibly its absolute quality. The model is a natural extension of Reference [27].

Agents. There are $n$ agents who compete in a contest. Each agent simultaneously chooses a level of effort $e_{i} \geq 0$ to exert. The quality $q_{i}$ of agent $i$ 's output is determined both by her effort, and a random noise term $\epsilon_{i}$ as $q_{i}=e_{i}+\epsilon_{i}$, where each $\epsilon_{i}$ is an independent and identically distributed draw from a cumulative distribution function $F(\cdot)$. The noise $\epsilon_{i}$ has a number of possible interpretations. Most simply, $\epsilon_{i}$ could model the fact that a given amount of effort does not deterministically guarantee a certain level of output, but rather only influences its expected value. As another interpretation, the noise $\epsilon$ could also model randomness in the measurement, or perception, of an agent's output quality by the principal. Most interestingly, $\epsilon_{i}$ could be thought of as modeling heterogeneity amongst agents' abilities to solve the specific problem or execute the specific task required for the contest; we discuss this in detail at the end of this section. We will assume throughout that the probability density function $f(\epsilon)$ corresponding to $F(\epsilon)$ is a bounded continuously differentiable function of $\epsilon$ with a bounded first derivative, and the distribution $F(\cdot)$ and the number of agents $n$ are known to all agents.

Utilities. An agent's utility is the difference between her benefit from any prize she wins and her cost of effort. An agent who receives a prize $A_{i}$ derives a benefit of $v\left(A_{i}\right)$, where $v(\cdot)$ is a strictly increasing and concave function satisfying $v(A) \geq 0$ for all $A \geq 0$ and $v(0)=0$. Exerting effort $e_{i}$ incurs a cost $c\left(e_{i}\right)$, where $c(\cdot)$ is a strictly increasing and convex function satisfying $c(e) \geq 0$ for all $e \geq 0$ and $c^{\prime}(0)=0 .{ }^{4}$ We begin our analysis by considering the case where $c(0)=0$, so that all agents have an incentive to participate in equilibrium; in Section 7, we extend our analysis to consider the case where $c(0)>0$ and agents make endogenous decisions about whether to participate.

The utility to an agent who exerts effort $e_{i}$ and receives prize $A_{i}$ is the difference between this benefit and cost, $u_{i}=v\left(A_{i}\right)-c\left(e_{i}\right)$. We assume that each agent chooses $e_{i}$ to maximize her expected utility $E\left[v\left(A_{i}\right)\right]-c\left(e_{i}\right)$, where the expectation is taken over the $n$ random draws of $\epsilon_{i^{\prime}}$ that determine each agent's output quality (and therefore their prizes). Our model allows for both risk-neutral and risk-averse agents, as $v(A)$ may either be a linear function of $A$ (for risk-neutral agents) or a strictly concave function of $A$ (risk-averse agents).

Mechanisms. We suppose that the principal running the contest can observe the quality $q_{i}$ of each agent's output. (Our results extend immediately to a model where the principal's observations of output qualities are noisy, because the noise can be folded into the $\epsilon_{i}$ term.) We use $\mathcal{M}\left(A_{1}, A_{2}, \ldots, A_{n}\right)$ to denote a rank-order mechanism that assigns a reward $A_{j}$ to the agent with the $j$ th-highest output regardless of its absolute quality, and assume throughout that $A_{1} \geq \cdots \geq A_{n} \geq 0$. $^{5}$

Mixed cardinal-ordinal mechanisms. Let $q_{j}$ denote the quality of the $j$ th-ranked submission. We consider mixed cardinal-ordinal modifications of a rank-order mechanism $\mathcal{M}\left(A_{1}, A_{2}, \ldots, A_{n}\right)$

\footnotetext{
${ }^{4}$ The assumption that $c^{\prime}(0)=0$ is primarily used to help prove equilibrium existence.

${ }^{5}$ It is worth noting, however, that most of the proofs and results in this article will continue to hold even if this assumption on the monotonicity of rewards is relaxed. In particular, the main result in our article about the optimality of threshold mechanisms will hold more generally.
} 
of the form $\mathcal{M}\left(g_{1}\left(q_{1}\right) A_{1}, \ldots, g_{n}\left(q_{n}\right) A_{n}\right)$, which awards the agent with the $j$ th-ranked submission of quality $q_{j}$ a prize $P_{j}=g_{j}\left(q_{j}\right) A_{j}$, where $g_{j}(q)$ is a non-decreasing function of $q$ satisfying $0 \leq$ $g_{j}(q) \leq 1$. That is, $g_{j}(q)$ represents the fraction of the maximum prize $A_{j}$ for achieving rank $j$ that an agent obtains if she produces a contribution with absolute quality $q$. Note that the rank-order mechanism $\mathcal{M}\left(A_{1}, A_{2}, \ldots, A_{n}\right)$ corresponds to setting $g_{j}(\cdot)$ to be the constant function $g_{j}(q)=1$ for all $j$.

We note that a principal with access to cardinal measurements of the qualities of each submission could conceivably use more general mechanisms by allowing the function $g_{j}(\cdot)$ to depend not only on the quality of the corresponding $j$ th-ranked submission, but rather on the entire vector of qualities $\left(q_{1}, \ldots, q_{n}\right)$. We restrict ourselves to mechanisms that use functions $g_{j}\left(q_{j}\right)$ for simplicity, both of analysis and implementation. In addition to leading to simpler mechanisms, a principal might, practically speaking, prefer to announce a contest where the prize awarded to a winner is contingent on the absolute quality of only her own submission, and not on the absolute qualities of the submissions produced by her competitors; see also the discussion in Section 1.

Principal's objective. We assume that the principal's objective is to maximize some utility function $W\left(q_{1}, \ldots, q_{n}\right)$ of agents' output qualities in equilibrium, where $W$ is non-decreasing in its arguments, i.e., $W\left(q_{1}, \ldots, q_{n}\right)$ is such that if $q_{i}^{\prime} \geq q_{i}$ for all $i$, then $W\left(q_{1}^{\prime}, \ldots, q_{n}^{\prime}\right) \geq W\left(q_{1}, \ldots, q_{n}\right)$. Implicitly, here we are focusing on a setting in which the principal has a fixed budget that represents the maximum amount the principal can spend, and the principal seeks to maximize some utility function of the quality of the agents' contributions subject to the constraint that the total prizes paid out must not exceed this budget. Such a setting has also been considered in several other papers in the contest design literature, such as References [8], [31], and [32]. It is a reasonable model of a middle manager who is given some budget to run a contest with the objective of obtaining the highest quality contributions possible, but does not keep any of the budget that is not spent. In this setting, the manager only cares about the quality of the contributions and does not care about the amount of prizes that are paid out (beyond needing to ensure that total prize payments are less than or equal to the budget), so the cost of the prizes does not directly enter the principal's utility, as in References [8], [31], and [32].

We will be interested throughout in symmetric pure-strategy Nash equilibria. If agents all use the same level of effort, then the principal's expected utility is non-decreasing in the effort choice of the agents: therefore, all such increasing objectives are simultaneously improved by mechanisms that elicit higher equilibrium effort, assuming a symmetric equilibrium.

Heterogeneity. Our model, with noise terms $\epsilon_{i}$ all drawn from the same distribution $F(\cdot)$ and costs to effort given by the same function $c_{i}(\cdot)=c(\cdot)$, suggests contributors who are $a$ priori homogeneous, corresponding to a pool of contestants who all have similar abilities for the subject of the contest (for example, programmers with similar levels of expertise or graphic designers with similar skill levels). Such a model captures scenarios where it is predominantly differences in effort, rather than differences in ability, that dominate differences in the quality produced. It also captures scenarios where the contestants may be self-selected to have rather similar abilities or expertise levels, and therefore similar costs to producing a particular quality.

Our model can nonetheless capture agent heterogeneity in two different ways. First, two contestants with the same effort choice will still come up with solutions of different qualities depending on their draws of $\epsilon_{i}$, corresponding to situations where agents with similar skills nonetheless produce different outputs for the specific task posed by a particular contest. Second, the incentives of agents in contests where there is indeed heterogeneity in abilities that affects agents' final output qualities, but where agents do not know these abilities (beyond their distribution) prior to making their strategic effort choices, are identical to those in our model with 'abilities' drawn from $F(\cdot)$ after the agents choose their effort levels. Therefore, this model of heterogeneity includes many 
situations with non-homogeneous contributors, as long as agents do not learn their abilities prior to choosing their effort levels.

In a different model of heterogeneity amongst agents that appears in the contest design literature, an agent's output $q_{i}$ is her ability-scaled effort $a_{i} e_{i}$, where agents' abilities are all randomly drawn from a single distribution $F(\cdot)$ : a logarithmic transformation of variables from $q_{i}=a_{i} e_{i}$ in those models yields exactly our model where $q_{i}=e_{i}+\epsilon_{i}$. The key difference between these two models of heterogeneity is timing of information-agents observe their random draws of $a_{i}$ before making their strategic effort choices in those models, whereas agents do not observe their draws of $\epsilon_{i}$ prior to making their effort choices in our model (for instance, as with a graphic designer who does not know exactly how good a design she will produce until she attempts it).

\section{OPTIMALITY OF THRESHOLD MECHANISMS}

A contestant's cardinal quality score can be incorporated into a given rank-order mechanism in many different ways. A mechanism might choose to increase the reward for each rank linearly with an agent's output; more generally, the reward might increase as some convex function of her cardinal quality score up to some maximum reward. The reward scheme could also vary discontinuously with the quality of the agent's output, for instance, so that the reward for a particular rank is determined by which of several intervals the corresponding quality score lies in. A priori, each of these cardinal modifications to a rank-order mechanism might create stronger incentives for effort than a purely ordinal mechanism by making rewards more strongly dependent on the absolute quality. What choice of functions $g_{j}(\cdot)$ creates the strongest incentives for effort amongst all non-decreasing $g_{j}(\cdot)$ ?

While analyzing this question, we make two simplifying assumptions. First, we consider functions $g_{j}(q)$ for which there is some small $\delta_{j}>0$ such that $g_{j}(q)$ may only assume values that are integral multiples of $\delta_{j}$. While this assumption is purely for technical simplicity and our results continue to hold without this assumption (albeit with a more complex proof), we note that this assumption is realistic, because in any practical application there will be some minimum unit of a currency that represents the smallest possible amount by which one can change the value of an agent's prize. (For example, if prizes were paid in U.S. dollars, any prize would necessarily have to be some integral multiple of some small fraction of a penny, as a principal would not be able to divide an agent's prize further than this). Second, we assume that there is a unique symmetric pure-strategy equilibrium in which all agents exert the same effort level $e$ in the game; ${ }^{6}$ we show in the appendix (Theorem A.1) that a unique symmetric pure-strategy equilibrium will exist under the assumption that a player's cost function is sufficiently convex. ${ }^{7}$

Our main result shows that the question of how to choose the functions $g_{j}(q)$ to optimally modify any given rank-order mechanism has a strikingly simple answer: For any given rank-order mechanism $\mathcal{M}\left(A_{1}, A_{2}, \ldots, A_{n}\right)$ with fixed $A_{1}, \ldots, A_{n}$, no other function can incentivize higher equilibrium effort than the optimal step functions that increase from 0 to 1 at some threshold score. We refer to such mechanisms as threshold mechanisms.

THEOREM 3.1. Suppose there exists a unique symmetric pure-strategy equilibrium in any rankorder tournament in which the agent who finishes in $j$ th place is a warded a prize $g_{j}(q) A_{j}$, where $g_{j}(q)$ is a non-decreasing function satisfying $0 \leq g_{j}(q) \leq 1$ for all $q$. Then there exist functions $g_{j}(q)$ of the

\footnotetext{
${ }^{6}$ In particular, we also assume that there is a unique value of $e$ that is a solution to Equation (4) in the Appendix, which characterizes the equilibrium level of effort.

${ }^{7}$ Furthermore, we also prove in Theorem A.3 that if there is enough noise in the sense that the noise terms $\epsilon_{i}$ are drawn from a distribution with sufficiently high variance, then only a minimal amount of convexity is needed to ensure existence of a unique symmetric pure-strategy equilibrium.
} 
form $g_{j}(q)=0$ for $q<q_{j}^{*}$ and $g_{j}(q)=1$ for $q \geq q_{j}^{*}$ for some constants $q_{j}^{*}$ that incentivize the highest equilibrium effort amongst all possible mechanisms characterized by some functions $g_{j}(q)$.

All proofs are in the Appendix.

While there are potentially a variety of more nuanced ways to incorporate cardinal information in determining agents' prizes, Theorem 3.1 says that there is always an optimal mechanism with an exceedingly simple form-it awards the entire value of the $j$ th prize to the agent who finishes in $j$ th place if this agent's output quality meets some threshold, and awards her no prize at all otherwise. This result may be surprising, since it would seem more natural to use a mechanism where an agent's prize varies smoothly with the quality of her output, especially if agents are riskaverse-risk-averse agents are likely to prefer a prize structure in which they have a good chance of receiving a moderate prize over one in which they have a high chance of receiving nothing and a high chance of receiving a large prize. Nonetheless, Theorem 3.1 shows that such threshold mechanisms can always incentivize agents to choose optimal effort levels.

The reason that Theorem 3.1 holds is as follows: If awarding an agent some fraction of the $j$ th prize for output of quality $q_{j}$ would create an incentive for an agent to exert more effort than not awarding the agent at all, then awarding the agent an even larger fraction of the $j$ th prize for output of quality $q_{j}$ would create an even stronger incentive to exert more effort. Thus, if it is not optimal to award the agent none of the $j$ th prize for output of quality $q_{j}$, it will be optimal to award the agent all of the $j$ th prize for output quality of $q_{j}$. However, it could be sensible to award the agent none of the $j$ th prize for low quality output, as this may create a disincentive for low absolute quality. This in turn implies that the optimal mechanism is a threshold mechanism.

It is also worth noting that this result will hold even if there is a common shock to the agents' output in the sense that $q_{i}=e_{i}+\epsilon_{i}+\eta$ for some randomly drawn $\eta$ that is common to all agents. A substantively identical proof can be used to prove Theorem 3.1 in this more general model. This addresses a natural question in regards to settings with observable outputs, namely why use a contest at all-the principal might directly write contracts with individuals that specify rewards contingent on absolute performance. In a setting in which there are common shocks to the agents' outputs, it is known that it is essential to use tournaments rather than a reward scheme based purely on an individual's output in optimal contracts [20]. Thus, the main result of our article about the optimal cardinal contests also holds in a setting in which it is necessary to use a contest structure to elicit optimal contributions.

\section{OPTIMAL THRESHOLDS}

Our main result shows that the optimal mechanism is a threshold mechanism; we now ask what the optimal thresholds are. The proof of optimality of threshold mechanisms does not say anything about how the thresholds corresponding to each $\operatorname{rank} j$ vary with $j$, and thus allows for the possibility that the optimal thresholds might differ substantially for each rank. However, while it might seem intuitive that the optimal threshold could either consistently increase or consistently decrease across ranks, this turns out not to be the case, as we show below:

Theorem 4.1. Suppose the noise density $f(\cdot)$ is single-peaked at $0 .{ }^{8}$ Then the optimal threshold mechanism $\mathcal{M}\left(A_{1}, t_{1}^{*}, \ldots, A_{n}, t_{n}^{*}\right)$ applies the same threshold to each rank $j$ for any monotone mechanism $\mathcal{M}\left(A_{1}, A_{2}, \ldots, A_{n}\right)$, i.e., $t_{j}^{*}=t^{*}$ for $j=1, \ldots, n$.

Theorem 4.1 says that optimally incorporating cardinal scores into a rank-order contest to maximize effort is, in fact, even simpler than the method suggested by Theorem 3.1-a mechanism

\footnotetext{
${ }^{8}$ Analogs of the results in this section and Section 7 can also be proven for noise densities that are single-peaked at an arbitrary value at the cost of complicating the exposition.
} 
designer only needs to compare all agents' outputs against the same baseline, reducing the problem of finding the optimal modification of $\mathcal{M}\left(A_{1}, A_{2}, \ldots, A_{n}\right)$ to one of choosing a single optimal threshold $t^{*}$ for $\mathcal{M}\left(A_{1}, A_{2}, \ldots, A_{n}\right)$. Thus, the optimal threshold mechanism would remove all agents whose quality falls below a certain threshold, and award prizes to the remaining contestants by using the original mechanism.

The intuition for this result is that if the mechanism designer used thresholds that decreased with rank, then it would be possible for the agent with the highest realized quality to actually receive a lower prize than an agent who finishes in second place, because the agent with the highest quality contribution failed to meet the threshold for the corresponding rank while the agent who finished second did not. This in turn would create an incentive for agents to potentially not want to finish in a higher place, thereby decreasing incentives to exert effort. It would also be unlikely for the optimal thresholds to increase with rank, thereby requiring higher absolute standards from lower-ranking agents than higher-ranking ones. Together, these arguments suggest that the optimal threshold mechanism should use the same thresholds for all ranks.

Our proof of Theorem 4.1 also contains a proof of the following result, which we state here, since it is used repeatedly in the remainder of our analysis:

Corollary 4.2. Suppose the noise density $f(\cdot)$ is single-peaked at 0. Then if $t^{*}$ denotes the optimal threshold and $e^{*}(t)$ denotes the equilibrium effort at a given threshold $t$, then $t^{*}=e^{*}\left(t^{*}\right)$.

While equilibrium effort is maximized at the optimal threshold $t^{*}$, a mechanism designer without access to precise information about the parameters of the population of contestants may not be able to compute and use the optimal threshold $t^{*}$ for this population. Our next result addresses the question of how the equilibrium effort $e^{*}(t)$ varies as a function of the threshold used to modify a mechanism $\mathcal{M}$ for arbitrary non-optimal thresholds $t$.

THEOREM 4.3. Suppose that $f(\cdot)$ is single-peaked at 0 . Then the equilibrium level of effort is greater than the threshold and increasing in the threshold for $t \leq t^{*}$ and lower than the threshold and decreasing in the threshold for $t>t^{*}$.

This result is relevant to the question of identifying the optimal threshold $t$ without complete knowledge of the contestant population when multiple iterations of a contest will be held with different agents. Suppose a mechanism designer uses some particular threshold $t$ and observes the qualities of the submissions elicited with that threshold. When the number of contestants $n$ is large, the mean of the elicited qualities $q_{i}=e^{*}(t)+\epsilon_{i}$ provides a reasonable estimate of the equilibrium effort $e^{*}(t)$. Thus, a principal who repeatedly runs such a contest would be able to make probabilistic inferences as to whether equilibrium effort $e^{*}(t)$ was higher or lower than the threshold $t$ that was used. The theorem above indicates how the mechanism designer can use his estimate of $e^{*}(t)$ to update the threshold for the next iteration of the contest: since equilibrium effort is both greater than the threshold and increasing in the threshold when $t<t^{*}$, if equilibrium effort was probably greater than the threshold, then the threshold $t$ is likely to be below the optimum so the principal should increase the threshold, and vice versa.

\subsection{Comparative Statics}

We now address the question of how the optimal threshold $t^{*}$ varies with changes in the number of contestants and the prize structure. First, we consider comparative statics with respect to the number of agents. Our mechanisms $\mathcal{M}\left(A_{1}, A_{2}, \ldots, A_{n}\right)$ so far have been specified in terms of the prizes for each of the $n$ ranks, where $n$ is the number of players. Since we want to now vary $n$, we 
assume that there is a fixed number $k$ of prizes, $A_{1}, A_{2}, \ldots, A_{k}$, and the number of players $n \geq k$. In this scenario, we prove the following result:

Theorem 4.4. Consider any given rank-order mechanism $\mathcal{M}$ with prizes $A_{1}, \ldots, A_{k}$, and let $t^{*}(n)$ denote the optimal threshold for $\mathcal{M}$ when there are $n$ agents in the contest. If the noise density $f(\cdot)$ is single-peaked at 0 , then the optimal threshold $t^{*}(n)$ is decreasing in the number of players $n$ for all $n \geq k$. Furthermore, the equilibrium effort $e^{*}\left(t^{*}(n)\right)$ in the optimal threshold mechanism also decreases with $n$.

To understand the reasoning behind Theorem 4.4, note that when there are more agents in the contest, the expected prize that any particular agent will be able to achieve will be smaller. Thus, agents have less incentive to exert effort when there are more agents in the contest, and equilibrium effort will be smaller when $n$ is larger. At the same time, since the optimal threshold is equal to equilibrium effort at that threshold, the optimal threshold is also decreasing in the number of players $n$. This explains the results in Theorem 4.4.

Theorem 4.4 illustrates how equilibrium effort varies with the number of players $n$, but it is worth noting that similar results can be obtained about how the average quality of the contributions varies with $n$. Since the average quality of the contributions is $\bar{q}=e^{*}\left(t^{*}(n)\right)+E\left[\epsilon_{i}\right]$ and $E\left[\epsilon_{i}\right]$ is independent of $n$, the result in Theorem 4.4 immediately implies that the average quality of the contributions is also decreasing in $n .^{9}$

Next, we ask how the optimal threshold varies with the number of prizes awarded. To formulate this question meaningfully, we consider contests where the top $k$ participants who meet the threshold all receive the same prize and consider two ways that the total number of prizes might increase-first, where the total prize pool stays the same, but the prizes are split amongst a larger number of players, and second, where the value of each prize stays the same, but more prizes are awarded (contingent on meeting the threshold). The optimal threshold varies predictably with these changes in the prize structure, as the following theorem illustrates:

Theorem 4.5. Suppose that $f(\cdot)$ is single-peaked at 0 and the number of prizes is less than the number of players.

(1) The optimal threshold $t^{*}$ in a contest with $k$ equal prizes of value $A$ is increasing in $k$.

(2) The optimal threshold $t^{*}$ in a contest with $k$ equal prizes of value $A / k$ is increasing in $k$ if players are sufficiently risk-averse. Formally, there is some $\alpha>0$ such that if the coefficient of absolute risk aversion, $-\frac{v^{\prime \prime}(\cdot)}{v^{\prime}(\cdot)}$, satisfies $-\frac{v^{\prime \prime}(A)}{v^{\prime}(A)} \geq \alpha$ for all $A$, then the optimal threshold $t^{*}$ in a contest of $k$ equal prizes of value $\frac{A}{k}$ is increasing in $k$.

The intuition for this result has to do with how an agent's incentives to try to meet the threshold vary with the number of prizes. As the number of prizes increases in either of the two manners considered in Theorem 4.5, the expected value of the prize that an agent obtains for meeting the threshold increases. Thus, agents have a stronger incentive to try to meet the threshold, and agents will thus exert more effort in equilibrium when there are a larger number of prizes. ${ }^{10}$ Since the optimal threshold is equal to the equilibrium level of effort, it then follows that increasing the number of prizes also increases the optimal threshold.

\footnotetext{
${ }^{9}$ However, there is no general result about how the average quality of the best contribution varies with $n$. The average quality of the best contribution is $q_{\max }=e^{*}\left(t^{*}(n)\right)+E\left[\max _{i}\left\{\epsilon_{i}\right\}\right]$. Since $e^{*}\left(t^{*}(n)\right)$ is decreasing in $n$ while $E\left[\max _{i}\left\{\epsilon_{i}\right\}\right]$ is increasing in $n$, there is no general result about how $q_{\max }$ varies with $n$.

${ }^{10}$ Similarly, the average quality of the contributions and the average quality of the best contribution will also increase when there are a larger number of prizes.
} 


\section{HOW USEFUL IS CARDINAL INFORMATION?}

Our results thus far show that making the award of the prizes in a rank-order contest $\mathcal{M}\left(A_{1}, A_{2}, \ldots, A_{n}\right)$ contingent on submission qualities exceeding a suitable threshold creates the strongest incentives for effort, and in particular, outperforms the contest that ignores cardinal information. In this section, we address the question of how much of an improvement is obtained from using this cardinal information relative to $\mathcal{M}\left(A_{1}, A_{2}, \ldots, A_{n}\right)$, which awards prizes based only on relative rankings.

We first present simulation results to obtain a sense of the size of the improvement in equilibrium effort as a function of the number of contestants, the nature of participants' cost functions, the reward structure $\left(A_{1}, A_{2}, \ldots, A_{n}\right)$, and the noise distribution governing the stochastic perturbations that influence an agent's output. We ask to what extent the size of these gains are affected by changes in these underlying parameters, and discuss these results in the context of typical parameter values in online contests. Finally, we conclude with a theorem that formalizes the observations from these simulations.

Simulations. We simulate contests where $n$ players each have a cost function $c(\hat{e})=\frac{\hat{e}^{a}}{a}$ for some constant $a>1$ (a larger $a$ corresponds to a more convex cost function). We assume that players are risk-neutral with values $v(A)=A$ for a prize $A$. For the distribution of the noise terms $\epsilon_{i}$ that randomly influence a player's submission quality as $q_{i}=e_{i}+\epsilon_{i}$, we consider IID draws from (i) a standard normal distribution and (ii) a standard Laplace distribution. For the set of prize structures, we consider contests with prizes of $A_{1}$ and $A_{2}$ for the top two ranks and $A_{j}=0$ for all lower ranks for simplicity and brevity. Each of $n, a$, and the split into $A_{1}$ and $A_{2}$ are parameters describing the contest that we will vary in our simulations.

Computing equilibrium efforts. To quantify the extent of improvement in equilibrium effort from using cardinal information in a contest, we first need to calculate the equilibrium level of effort both with no threshold and with the optimal threshold. When there is no threshold, the equilibrium level of effort is the same as it would be if the threshold were $t=-\infty$. Applying Equation (7) in the Appendix to the special case in which $t=-\infty$, we see that the equilibrium level of effort $e_{\phi}^{*}$ with no thresholds must satisfy

$$
\begin{aligned}
e_{\phi}^{* a-1}= & \sum_{j=1}^{n} v\left(A_{j}\right)\left[\int _ { - \infty } ^ { \infty } \left[\frac{(n-1) !}{(j-1) !(n-j-1) !}\left(1-F\left(\epsilon_{i}\right)\right)^{j-1} F\left(\epsilon_{i}\right)^{n-j-1}\right.\right. \\
& \left.\left.-\frac{(n-1) !}{(j-2) !(n-j) !}\left(1-F\left(\epsilon_{i}\right)\right)^{j-2} F\left(\epsilon_{i}\right)^{n-j}\right] f^{2}\left(\epsilon_{i}\right) d \epsilon_{i}\right]
\end{aligned}
$$

where we abuse notation by defining $\frac{(n-1) !}{(j-2) !(n-j) !}$ to be zero when $j=1$.

When the mechanism designer uses the optimal threshold $t^{*}$, we know from Corollary 4.2 that the equilibrium level of effort $e_{O}^{*}$ equals the optimal threshold. Setting $t=e$ in Equation (7), we see that $e_{O}^{*}$ must satisfy

$$
\begin{aligned}
e_{O}^{* a-1}=\sum_{j=1}^{n} v\left(A_{j}\right)\left[\left(\begin{array}{c}
n-1 \\
j-1
\end{array}\right)\left(\frac{1}{2}\right)^{n-1} f(0)\right. & +\int_{0}^{\infty}\left[\frac{(n-1) !}{(j-1) !(n-j-1) !}\left(1-F\left(\epsilon_{i}\right)\right)^{j-1} F\left(\epsilon_{i}\right)^{n-j-1}\right. \\
& \left.\left.-\frac{(n-1) !}{(j-2) !(n-j) !}\left(1-F\left(\epsilon_{i}\right)\right)^{j-2} F\left(\epsilon_{i}\right)^{n-j}\right] f^{2}\left(\epsilon_{i}\right) d \epsilon_{i}\right] .
\end{aligned}
$$

We can compute the percentage increase in equilibrium effort for a particular set of contest parameters by using the equations above to compute $e_{\phi}^{*}$ and $e_{O}^{*}$. We compute these improvements for a wide range of different parameters to observe how the various parameters affect the extent 
Table 1. Percentage Increase in Equilibrium Effort from Using the Optimal Threshold Under a Winner-take-all Contest When the Cost to Effort $\hat{e}$ is $c(\hat{e})=\frac{\hat{e}^{2}}{2}$ for Different Noise Distributions and Varying Values of the Number of Players $n$

\begin{tabular}{|c|c|c|}
\hline$n$ & Normal distribution & Laplace distribution \\
\hline 2 & $20.71 \%$ & $50.00 \%$ \\
\hline 3 & $4.95 \%$ & $16.67 \%$ \\
\hline 4 & $1.59 \%$ & $7.14 \%$ \\
\hline 5 & $0.58 \%$ & $3.33 \%$ \\
\hline 6 & $0.23 \%$ & $1.61 \%$ \\
\hline 7 & $0.10 \%$ & $0.80 \%$ \\
\hline 8 & $0.04 \%$ & $0.40 \%$ \\
\hline 9 & $0.02 \%$ & $0.19 \%$ \\
\hline
\end{tabular}

Table 2. Percentage Increase in Equilibrium Effort from Using the Optimal Threshold for Different Noise Distributions and Varying Values of the Term $a$ in the Cost Function $c(\hat{e})=\frac{\hat{e}^{a}}{a}$, Under a Winner-take-all Contest with $n=4$

\begin{tabular}{|c|c|c|}
\hline$a$ & Normal distribution & Laplace distribution \\
\hline 1.25 & $6.51 \%$ & $31.78 \%$ \\
\hline 1.5 & $3.21 \%$ & $14.80 \%$ \\
\hline 1.75 & $2.12 \%$ & $9.64 \%$ \\
\hline 2 & $1.59 \%$ & $7.14 \%$ \\
\hline 3 & $0.79 \%$ & $3.51 \%$ \\
\hline 4 & $0.53 \%$ & $2.33 \%$ \\
\hline 5 & $0.40 \%$ & $1.74 \%$ \\
\hline 6 & $0.32 \%$ & $1.39 \%$ \\
\hline
\end{tabular}

Table 3. Percentage Increase in Equilibrium Effort from Using the Optimal Threshold for Different Noise Distributions and Varying Distributions of the Top Prizes, with Five Players When $c(\hat{e})=\frac{\hat{e}^{2}}{2}$

\begin{tabular}{|c|c|c|}
\hline$\left(A_{1}, A_{2}\right)$ & Normal distribution & Laplace distribution \\
\hline$(1,0)$ & $0.58 \%$ & $3.33 \%$ \\
\hline$(0.9,0.1)$ & $1.06 \%$ & $5.17 \%$ \\
\hline$(0.8,0.2)$ & $1.59 \%$ & $7.14 \%$ \\
\hline$(0.7,0.3)$ & $2.20 \%$ & $9.26 \%$ \\
\hline$(0.6,0.4)$ & $2.90 \%$ & $11.54 \%$ \\
\hline$(0.5,0.5)$ & $3.72 \%$ & $14.00 \%$ \\
\hline
\end{tabular}

of the increase in equilibrium effort from using cardinal information via the optimal threshold mechanisms. The results are summarized in Tables 1-3.

Discussion of simulation results. The simulations lead to a number of interesting insights. First, they show that when the number of players is larger, the benefit to setting the optimal threshold is relatively smaller. We also see that when the parameter $a$ in the exponent of the cost function $c(\hat{e})=\frac{\hat{e}^{a}}{a}$ increases, so that players' cost functions are more convex, less is gained by using the 
optimal threshold. Finally, when more of the prizes are given to the lower-ranked players, there is again a larger benefit from using a threshold.

These observations all have intuitive explanations. Since equilibrium effort equals the optimal threshold (Corollary 4.2), when there are a large number of players, the winning players' final realized quality is likely to far exceed the threshold, so the threshold provides little incentive for effort. By contrast, when there are only a small number of players, there is a significant risk that even the winning player's final realized quality will be in the same range as the threshold, and the threshold may therefore provide significant incentives to exert effort. Thus, using the optimal threshold should have a relatively smaller effect when there are a larger number of players.

We note that both regimes with large numbers of players and small numbers of players are relevant empirically. For example, in Kaggle contests, there are typically at least dozens and usually hundreds of participants, so in such a contest, setting the optimal threshold is likely to have little effect on incentives to exert effort. By contrast, many tasks on TaskCN and Topcoder ${ }^{11}$ only attract a few participants, ${ }^{12}$ so in such contests, using the optimal threshold is likely to significantly improve effort. There are also online contests with elimination rounds leading up to a final round with a few finalists (such as the Robin Hood Foundation Prize ${ }^{13}$ ). In such settings, our results would suggest that using an optimal threshold to award prizes is likely to significantly improve effort in the final round of the contest.

To understand why convexity affects equilibrium effort, note that when the players' cost functions are more convex, a change in a player's effort does relatively more to increase the player's marginal cost of exerting effort, which in turn implies that a player can only increase her effort by a smaller amount in response to improved incentives before the player's marginal cost from exerting more effort will equal her marginal benefit. Thus, using the optimal threshold will have less effect when the players' cost functions are more convex.

Finally, we address the effect of the distribution of prizes. The lower-ranked players are more likely to have final realized qualities that are close to the threshold, so the threshold provides a greater incentive for a lower-ranked player to exert effort than a higher-ranked player. Thus, when a greater amount of the prize pool is given to the lower-ranked players, this shifts prizes from players who will be less strongly incentivized by the thresholds to players who will be more strongly incentivized by the thresholds. Thus, using the optimal threshold has a relatively greater effect when a larger amount of the prize pool is distributed amongst lower ranks.

All these insights are, in fact, more general than this, as shown below:

THEOREM 5.1. Consider values of the number of players $n$ for which there exists a unique symmetric pure-strategy equilibrium. ${ }^{14}$ Let $e_{\text {opt }}$ denote the equilibrium effort that is achieved under the optimal threshold and let $e_{0}$ denote the equilibrium effort that is achieved when there is no threshold. Then, we have the following results:

(1) If $c(e)=\frac{e^{2}}{2}, f(\cdot)$ is symmetric about 0 , and the number of players $n$ is at least twice as large as the number of prizes $k$, then the difference $e_{o p t}-e_{0}$ is decreasing in $n$.

\footnotetext{
${ }^{11}$ http://community.topcoder.com/tc?module=CompList\&pt $=35$.

${ }^{12}$ For example, Reference [29] notes that most programming tasks on TaskCN attracted fewer than six participants and [50] notes that it is far more common to receive a small number of solutions for a task on TaskCN than some larger number of solutions.

${ }^{13}$ https://www.robinhood.org/prize.

${ }^{14} \mathrm{An}$ immediate implication of Theorem A.3 is that if the noise terms $\epsilon_{i}$ are drawn from a distribution with sufficiently high variance, then a unique symmetric pure-strategy equilibrium will exist for a wide range of values of $n$.
} 
(2) If $c(e)=\frac{e^{2}}{2}, f(\cdot)$ is symmetric and single-peaked about 0 , the number of players $n$ is at least twice as large as the number of prizes $k$, and all prizes are equal, then the relative gain in equilibrium effort from the optimal threshold, $\frac{e_{o p t}-e_{0}}{e_{0}}$, is decreasing in $n$.

(3) If $c(e)=\frac{e^{2}}{2}, f(\cdot)$ is symmetric about 0 , the number of players $n$ is at least twice as large as the number of prizes $k$, and the prizes are all equal, then the difference $e_{o p t}-e_{0}$ is increasing in $k$.

(4) If $c(e)=\frac{e^{2}}{2}, f(\cdot)$ is symmetric and single-peaked about 0 , the number of players $n$ is at least twice as large as the number of prizes $k$, and the prizes are all equal, then the relative gain in equilibrium effort from the optimal threshold, $\frac{e_{o p t}-e_{0}}{e_{0}}$, is increasing in $k$.

(5) If $c_{2}(\cdot)$ is a more convex cost function than $c_{1}(\cdot)$ in the sense that the ratio $\frac{c^{\prime \prime}(e)}{c^{\prime}(e)}$ is greater for $c=c_{2}(\cdot)$ than it is for $c=c_{1}(\cdot)$ and the values of $e_{0}$ are equal under $c_{1}(\cdot)$ and $c_{2}(\cdot)$, then both the difference $e_{o p t}-e_{0}$ and the ratio $\frac{e_{o p t}-e_{0}}{e_{0}}$ are larger for $c_{1}(\cdot)$ than for $c_{2}(\cdot)$.

The results in this section so far have addressed how the increase in equilibrium effort from using the optimal threshold varies with the parameters, but one could also derive similar conclusions about other natural metrics of interest, such as the average quality of the contributions or the average quality of the best contribution. First, note that when $f(\cdot)$ is symmetric about zero, then the average quality of the contributions is just equal to the equilibrium level of effort $e$. Thus, all of our results in Theorem 5.1 about how the increase in equilibrium effort from using the optimal threshold varies with the parameters also apply to how the increase in the average quality of the contributions varies with the parameters.

These results also apply to how the increase in the average quality of the best contribution from using the optimal threshold varies with the parameters. Note that the average quality of the best contribution under a symmetric pure-strategy equilibrium will be $q_{\max }=e+E\left[\max _{i}\left\{\epsilon_{i}\right\}\right]$, where $e$ denotes equilibrium effort and $E\left[\max _{i}\left\{\epsilon_{i}\right\}\right]$ denotes the expectation of the largest value of $\epsilon_{i}$. Since $E\left[\max _{i}\left\{\epsilon_{i}\right\}\right]$ does not vary with the cost function or the number of prizes, any results in Theorem 5.1 about how the increase in equilibrium effort varies with the cost function or the numbers of prizes also apply to how the increase in the average quality of the best contribution varies with these parameters.

Similarly, the result in Theorem 5.1(i) about how $e_{o p t}-e_{0}$ is decreasing in the number of players $n$ implies that $q_{o p t}-q_{0}$ is decreasing in $n$, where $q_{o p t}$ denotes the average quality of the best contribution under the optimal threshold, and $q_{0}$ denotes the average quality of the best contribution under no threshold. And since $E\left[\max _{i}\left\{\epsilon_{i}\right\}\right]$ is increasing in $n$, the result in Theorem 5.1(ii) that $\frac{e_{o p t}-e_{0}}{e_{0}}$ is decreasing in $n$ also implies that $\frac{q_{o p t}-q_{0}}{q_{0}}=\frac{e_{o p t}-e_{0}}{e_{0}+E\left[\max _{i}\left\{\epsilon_{i}\right\}\right]}$ is decreasing in $n$. Thus, all the results in Theorem 5.1 about how the impact of the optimal threshold on equilibrium effort varies with the parameters also apply to how the increase in the average quality of the best contribution varies with the parameters.

Finally, we can also make statements about how the amount of surplus extracted by using the optimal threshold varies with the underlying parameters, where we define the amount of surplus extracted by the optimal threshold as the percentage decrease in the net utility of the players as a result of using the optimal threshold rather than no threshold. We first note how this surplus extracted varies with the number of players and the number of prizes in the same settings considered in Tables 1 and 3.

The results in Tables 4 and 5 suggest that the fraction of surplus extracted is larger both when the number of players $n$ is smaller and when more of the prize pool is given to the lower-ranked players. These results have intuitive explanations. 
Table 4. Percentage of Surplus Extracted from Using the Optimal Threshold (i.e., the Percentage Decrease in the Net Utility of the Players as a Result of Using the Optimal Threshold) Under a Winner-Take-all Contest When $c(\hat{e})=\frac{\hat{e}^{2}}{2}$ for different Noise Distributions and Varying Values of the Number of Players $n$

\begin{tabular}{|c|c|c|}
\hline$n$ & Normal distribution & Laplace distribution \\
\hline 2 & $31.11 \%$ & $46.43 \%$ \\
\hline 3 & $15.57 \%$ & $23.72 \%$ \\
\hline 4 & $7.69 \%$ & $11.23 \%$ \\
\hline 5 & $3.80 \%$ & $5.24 \%$ \\
\hline 6 & $1.87 \%$ & $2.45 \%$ \\
\hline 7 & $0.93 \%$ & $1.16 \%$ \\
\hline 8 & $0.46 \%$ & $0.56 \%$ \\
\hline 9 & $0.23 \%$ & $0.27 \%$ \\
\hline
\end{tabular}

Table 5. Percentage of Surplus Extracted from Using the Optimal Threshold (i.e., the Percentage Decrease in the Net Utility of the Players as a Result of Using the Optimal Threshold) for Different Noise Distributions and Varying Distributions of the Top Prizes, with Five Players When $c(\hat{e})=\frac{\hat{e}^{2}}{2}$

\begin{tabular}{|c|c|c|}
\hline$\left(A_{1}, A_{2}\right)$ & Normal distribution & Laplace distribution \\
\hline$(1,0)$ & $3.79 \%$ & $5.24 \%$ \\
\hline$(0.9,0.1)$ & $5.62 \%$ & $7.69 \%$ \\
\hline$(0.8,0.2)$ & $7.37 \%$ & $10.06 \%$ \\
\hline$(0.7,0.3)$ & $9.07 \%$ & $12.33 \%$ \\
\hline$(0.6,0.4)$ & $10.71 \%$ & $14.51 \%$ \\
\hline$(0.5,0.5)$ & $12.30 \%$ & $16.62 \%$ \\
\hline
\end{tabular}

When the number of players $n$ is larger, the fact that there is a smaller percentage difference in effort under no threshold and the optimal threshold also implies that there is a smaller percentage difference in cost of effort under no threshold and the optimal threshold. In addition, there is also a smaller percentage difference in the probability the prize will be paid out for the following reason: When there is no threshold, the probability the highest-ranked player's contribution will meet the threshold is 1 . By contrast, under the optimal threshold $t^{*}$, we know that $e^{*}\left(t^{*}\right)=t^{*}$ in equilibrium, so the probability any given player's contribution meets the threshold is just the probability that $\epsilon_{i} \geq 0$ or $F(0)=\frac{1}{2}$, meaning the probability the highest-ranked player's contribution will fail to meet the threshold is $\frac{1}{2^{n}}$, which is decreasing in $n$. Thus, when the number of players is larger, there is a smaller difference between the probability the prize will be paid out under the optimal threshold and under no threshold.

But since there is a smaller percentage difference in cost of effort and a smaller difference between the probability the winning prize will be paid out under no threshold and the optimal threshold when the number of players $n$ is larger, it is reasonable to also expect there to be a smaller percentage difference in the net utility of the players when the number of players $n$ is larger. Thus, the results in Table 4 make intuitive sense.

Similarly, when more of the prize pool is given to lower-ranked players, the fact that there will then be a larger percentage difference in effort under no threshold and the optimal threshold also 
implies that there is a larger percentage difference in cost of effort under no threshold and the optimal threshold. And since there is a larger probability that the lower-ranked players will fail to meet the optimal threshold than there is that the higher-ranked players will fail to meet the optimal threshold, the expected difference in the total value of the prizes paid out will be larger when more of the prize pool is given to lower-ranked players. Thus, it is also reasonable to expect there to be a larger difference in the net utility of the players when more of the prize pool is given to lower-ranked players, as we saw in Table 5.

\section{OPTIMAL PRIZE STRUCTURES}

In the previous section, we have seen that the size of the improvement in effort that can be achieved by using the optimal threshold can depend significantly on the parameters of the contest, including how the prize pool is divided amongst the various places. Various papers in the literature have addressed questions related to the optimal division of prizes in rank-order tournaments, but none have addressed this question in the context of mixed cardinal-ordinal tournaments. Would the optimal division of prizes change as a result of optimally incorporating cardinal information into a contest?

In particular, suppose the optimal division of the prize pool $A$ amongst the $n$ places for a rankorder mechanism is $\left(A_{1}, \ldots, A_{n}\right)$, where $A_{1} \geq \cdots \geq A_{n}$. Is it still the case that this is the optimal division of the prizes amongst the $n$ places for the optimal mixed cardinal-ordinal mechanism that makes use of the optimal threshold? In this section, we illustrate that the answer to this question depends crucially on whether the agents are risk-averse:

THEOREM 6.1. (1) If $v(A)=A$, then regardless of whether no threshold or the optimal threshold is used, the optimal prize structure is a winner-take-all prize structure. ${ }^{15}$

(2) If $v(\cdot)$ is strictly concave, then the optimal prize structures generally differ when the mechanism designer uses the optimal threshold instead of no threshold. In particular, if $n \geq 4$ and the prize pool is split between the top two places, then it is optimal to give a larger percentage of the prize pool to the second place finisher under the optimal threshold than under no threshold.

The intuition behind this result is as follows. We have seen that making use of the optimal threshold does more to increase the amount of effort agents exert in equilibrium when a larger fraction of the prize pool is given to the lower-ranked players. And since making use of the optimal threshold mechanism is more beneficial when a larger fraction of the prize pool is given to the lower-ranked players, the principal will generally have an incentive to give a larger percentage of the prize pool to the lower-ranked players when the principal is using the optimal threshold than under a pure rank-order mechanism. ${ }^{16}$

The only exception to this is when there is a non-interior solution to the optimal division of prizes, as when agents are risk-neutral. In this case, the same division of the prize pool may be optimal regardless of whether the principal uses the optimal threshold mechanism or a purely ordinal mechanism. Thus, the winner-take-all prize structure is optimal for risk-neutral agents regardless of whether the mechanism designer uses the optimal threshold.

\footnotetext{
${ }^{15}$ References [25] and [31] present similar results about the optimality of winner-take-all prize structures with no threshold, but do not prove such a result for threshold mechanisms.

${ }^{16}$ However, the principal will still typically have an incentive to give a larger fraction of the prize pool to the winner of the contest than the second-place finisher, even under the optimal threshold mechanism. This contrasts with Reference [30], which illustrates circumstances, albeit in a completely different model of contests, under which it may actually be optimal to give a larger fraction of the prize pool to the second-place finisher than to the winner of the contest.
} 


\section{ENDOGENOUS ENTRY}

In this section, we investigate cardinal contests with endogenous entry. We extend our model in Section 2 to incorporate endogenous entry as follows. Agents first simultaneously decide whether to participate in the contest; an agent who does not participate incurs no cost but also receives no prize, obtaining a utility of zero. An agent who does participate incurs a cost of effort, and an expected benefit depending on her performance in the contest; her final utility is exactly as in the model with exogenous entry in Section 2. After agents make their simultaneous participation decisions, each agent observes how many other agents have chosen to participate in the contest, and the agents then simultaneously decide how much effort to exert in the contest. We note that in this model with endogenous entry, agents' cost functions $c(\cdot)$ can be nonzero at 0 (i.e., $c(0)>0$ ), meaning that participating in the contest is allowed (though not required) to be strictly more costly than not participating at all, even if one exerts almost no effort.

A set of participation decisions is an equilibrium if no agent can profitably deviate by making a different participation choice, accounting for how the remaining participants will update their effort choice to account for this change in participation. Specifically, suppose $k$ agents decide to participate in a given threshold mechanism with threshold $t$, and let $e^{*}(t, k)$ denote the equilibrium level of effort in the game where exactly $k$ agents participate. For $k$ to be an equilibrium level of participation, (i) the expected utility to each of these $k$ agents when they participate with effort $e^{*}(t, k)$ and the remaining agents do not participate must be nonnegative, and (ii) none of the $n-k$ non-participants can profitably deviate by participating and choosing any effort level $e \geq 0$ when these $k$ agents are participating with effort $e^{*}(t, k+1)$. Throughout, we let $n$ denote the number of agents who must decide whether to participate in the contest and $k$ denote the number of agents who actually choose to participate in the contest; i.e., $n$ is the number of potential contestants and $k$ is the number of actual participants. We first note that there exists an equilibrium to the endogenous entry game under threshold mechanisms:

Theorem 7.1. Suppose entry is endogenous. For every threshold mechanism, there is a purestrategy equilibrium in which some agents participate with certainty and the remaining agents do not participate; furthermore, all participating agents choose the same level of effort.

We now investigate the nature of optimal thresholds. In general, different thresholds may result in different levels of participation in equilibrium, and the principal will face a tradeoff between the equilibrium number of participants and their level of effort. In the following theorem, we address the question of how a principal who desires a certain level of participation can choose the optimal threshold that maximizes effort from these participants. Let $t^{*}(k)$ denote the optimal threshold when exactly $k$ agents participate.

THEOREM 7.2. Suppose that $f(\cdot)$ is single-peaked at 0 , and $k$ is such that there exists some threshold $t$ such that it is an equilibrium for exactly $k$ agents to participate in the mechanism $\left(A_{1}, A_{2}, \ldots, A_{n}\right)$ modified by the threshold $t$. To maximize equilibrium effort subject to the constraint that exactly $k$ agents participate, the principal chooses the largest threshold less than or equal to $t^{*}(k)$ at which there is an equilibrium in which exactly $k$ agents participate.

Theorem 7.2 indicates that it will never be optimal for the principal to use a threshold greater than $t^{*}(k)$ to maximize equilibrium effort if he wants exactly $k$ agents to participate. We now illustrate how equilibrium participation levels vary with the threshold $t$ for such $t \leq t^{*}(k)$.

Theorem 7.3. Suppose that $f(\cdot)$ is single-peaked at 0 . Fix a rank-order mechanism $\mathcal{M}\left(A_{1}, A_{2}, \ldots, A_{n}\right)$, and restrict attention to thresholds $t \leq t^{*}(k)$, where $k$ denotes the equilibrium number of participants. Suppose that at the threshold $t_{1}$, there exists an equilibrium in which exactly 
$k_{1}$ agents participate. Then for all thresholds $t_{2} \leq t_{1}$, there exists some equilibrium in which exactly $k_{2}$ agents participate for some $k_{2} \geq k_{1}$.

Participation-effort tradeoffs. In general, different thresholds could lead to different equilibrium participation levels, since Theorem 7.3 indicates that equilibrium participation may increase as a result of decreasing the threshold. These equilibria with lower thresholds and higher participation will also lead to lower levels of effort-from Theorem 4.4, equilibrium effort decreases in the number of contestants, and from Theorem 4.3, equilibrium effort is increasing in the threshold for thresholds $t \leq t^{*}(k)$ (by Theorem 7.2, these are the only thresholds that the principal should consider under endogenous entry). Thus, while the principal will generally have several feasible levels of participation that he can induce by appropriately choosing the threshold, he will also face a participation-effort tradeoff-he can induce higher levels of participation by choosing a lower threshold, but this will come at the cost of lower equilibrium effort. The choice of which point to choose in the participation-effort tradeoff curve, and consequently the value of the optimal threshold, will be determined by how the principal values participation versus effort, which in turn can depend strongly on the context in which the contest is being held. ${ }^{17}$

\section{CONCLUSION}

In this article, we addressed the problem of how a principal running a contest might optimally incorporate cardinal information regarding the absolute qualities of contestants' entries into an existing rank-order tournament. We found that threshold mechanisms, which compare a submission's score against an absolute threshold-in fact, the same threshold for each prize-are optimal amongst the class of all mixed cardinal-ordinal mechanisms where the agent with the $j$ th-ranked submission with quality $q_{j}$ obtains an arbitrary quality-dependent fraction $g_{j}\left(q_{j}\right)$ of the prize $A_{j}$. Therefore, using cardinal information by comparing against a single threshold provides the optimal modification of a rank-order mechanism for incentivizing effort. Finally, we saw that the gains from incorporating cardinal information are highest for small contests with more than one prize and with participants whose costs to effort are not too convex.

A number of open questions remain for further research. First, the specific question we ask about modifying given rank-order mechanisms to incorporate cardinal information is motivated by the fact that practical considerations beyond incentives for effort-such as simplicity, sponsorships of various prize levels, media or publicity considerations-might cause a principal to choose a particular prize structure for his contest. However, it is also interesting to study the more general optimal contest design problem in such contests with access to absolute measurements of quality. What mechanism $\mathcal{M}\left(q_{1}, \ldots, q_{n}\right)$ incentivizes the highest effort over all mechanisms with access to cardinal, and not just ordinal, information about outputs? ${ }^{18}$ And how does the answer to this question depend on the specifics of the model such as agents' risk preferences and the objective function of the mechanism designer?

A second intriguing question regards a connection to optimal auctions and reserve pricing. There is a formal connection between auctions and contests $[8,9,12]$ in an alternative model for contests where effort deterministically translates into output. At first glance, this might suggest a

\footnotetext{
${ }^{17}$ It is worth noting that the equilibrium in Theorem 7.1 is not necessarily the only equilibrium, as there may also be a symmetric mixed-strategy equilibrium in which all agents participate with the same probability in equilibrium. Analyzing such an equilibrium would result in identical substantive conclusions to those in Theorem 7.3. Choosing a lower threshold will increase the probability with which agents participate in equilibrium, while decreasing the level of effort that such agents exert in equilibrium.

${ }^{18}$ Reference [8] addresses this question in a specific (but different) model; see Section 1.1.
} 
mapping between the analysis in our article and that in the literature on sponsored search auctions $[13,37]$. However, this mapping is a fallacious analogy in contests where output is a stochastic perturbation of effort, because the fact that an agent's output is determined by a noise-perturbed version of effort completely changes both the structure of the problem and all the underlying analysis relative to auctions. ${ }^{19}$ Nevertheless, the parallels between our results on optimality of thresholds in contests and auctions raise the question of whether there is a way to formally relate our results on contests to the optimality of reserve prices in auctions. A deeper understanding of the connection between threshold mechanisms and optimal auctions is an interesting direction for further research.

\section{APPENDIX}

\section{A PROOFS OF MAIN RESULTS}

\section{A.1 Equilibrium Existence}

Theorem A.1. There exists some constant $C=C\left(n, A_{j}, F\right)$ independent of the functions $g_{j}\left(q_{j}\right)$ such that if $c^{\prime \prime}(\cdot)>C$, then for any mixed-cardinal ordinal mechanism, there is a unique symmetric purestrategy equilibrium. ${ }^{20}$

Proof. Define a ladder function $l_{j}(q)$ to be a function characterized by a set of $m_{j}$ cutoffs $q_{1, j}^{*}<$ $q_{2, j}^{*}<\cdots<q_{m_{j}, j}^{*}$ such that $l_{j}(q)=r_{0, j}$ for $q<q_{1, j}^{*}, l_{j}(q)=r_{m, j}$ for all $q \in\left[q_{m, j}^{*}, q_{m+1, j}^{*}\right)$, and $l_{j}(q)=$ $r_{m_{j}, j}$ for $q \geq q_{m_{j}, j}^{*}$, where $0 \leq r_{0, j}<r_{1, j}<\cdots<r_{m_{j}, j} \leq 1$. Note that any non-decreasing function $g_{j}(q)$ such that $g_{j}(q)$ is an integral multiple of $\delta_{j}$ and $0 \leq g_{j}(q) \leq 1$ for all $q$ can be written in this form. Thus, to prove that a symmetric pure-strategy equilibrium exists and is unique for any mixed-cardinal ordinal mechanism, it suffices to show that such an equilibrium exists and is unique amongst the set of all mechanisms induced by ladder functions.

Let $y_{j}\left(e, e_{i}, \epsilon_{i}\right)$ denote the probability that agent $i$ finishes in $j$ th place for a given realization of $\epsilon_{i}$ given that agent $i$ exerts effort $e_{i}$ and all other agents exert effort $e$. If the prize for being ranked in $j$ th place with a contribution of quality $q$ is $l_{j}(q) A_{j}$ for some ladder function $l_{j}(q)$, then an agent $i$ 's expected utility from exerting effort $e_{i}$ when all other agents are exerting effort $e$ is

$$
\begin{aligned}
E\left[u_{i}\right] & =\sum_{j=1}^{n} \int_{-\infty}^{\infty} v\left(g_{j}\left(e_{i}+\epsilon_{i}\right) A_{j}\right) y_{j}\left(e, e_{i}, \epsilon_{i}\right) f\left(\epsilon_{i}\right) d \epsilon_{i}-c\left(e_{i}\right) \\
& =\sum_{j=1}^{n} \sum_{k=0}^{m_{j}} \int_{q_{k, j}^{*}-e_{i}}^{q_{k+1, j}^{*}-e_{i}} v\left(r_{k, j} A\right) y_{j}\left(e, e_{i}, \epsilon_{i}\right) f\left(\epsilon_{i}\right) d \epsilon_{i}-c\left(e_{i}\right),
\end{aligned}
$$

where we abuse notation by letting $q_{0, j}^{*} \equiv-\infty$ and $q_{m_{j}+1, j}^{*} \equiv \infty$. From this it follows that the derivative of the agent's utility with respect to $e_{i}$ is given by

$$
\begin{array}{r}
\sum_{j=1}^{n} \sum_{k=0}^{m_{j}} v\left(r_{k, j} A\right)\left[y_{j}\left(e, e_{i}, q_{k, j}^{*}-e_{i}\right) f\left(q_{k, j}^{*}-e_{i}\right)-y_{j}\left(e, e_{i}, q_{k+1, j}^{*}-e_{i}\right) f\left(q_{k+1, j}^{*}-e_{i}\right)\right. \\
\left.+\int_{q_{k, j}^{*}-e_{i}}^{q_{k+1, j}^{*}-e_{i}} \frac{\partial y_{j}\left(e, e_{i}, \epsilon_{i}\right)}{\partial e_{i}} f\left(\epsilon_{i}\right) d \epsilon_{i}\right]-c^{\prime}\left(e_{i}\right) .
\end{array}
$$

\footnotetext{
${ }^{19}$ Note that this is true even if we do not restrict attention to mechanisms that modify a particular rank-order mechanism in the manner we have considered in our article.

${ }^{20}$ One can prove that a symmetric equilibrium will always exist by applying the general equilibrium existence result in Reference [3]. However, if the cost function does not satisfy the condition in Theorem A.1, it may be that any symmetric equilibrium is in mixed strategies.
} 
Similarly, the second derivative of the agent's utility with respect to $e_{i}$ is

$$
\begin{array}{r}
\sum_{j=1}^{n} \sum_{k=0}^{m_{j}} v\left(r_{k, j} A\right)\left[\frac{\partial\left[y_{j}\left(e, e_{i}, q_{k, j}^{*}-e_{i}\right)\right]}{\partial e_{i}} f\left(q_{k, j}^{*}-e_{i}\right)-y_{j}\left(e, e_{i}, q_{k, j}^{*}-e_{i}\right) f^{\prime}\left(q_{k, j}^{*}-e_{i}\right)\right. \\
-\frac{\partial\left[y_{j}\left(e, e_{i}, q_{k+1, j}^{*}-e_{i}\right)\right]}{\partial e_{i}} f\left(q_{k+1, j}^{*}-e_{i}\right)+y_{j}\left(e, e_{i}, q_{k+1, j}^{*}-e_{i}\right) f^{\prime}\left(q_{k+1, j}^{*}-e_{i}\right) \\
+\left.\frac{\partial y_{j}\left(e, e_{i}, \epsilon_{i}\right)}{\partial e_{i}}\right|_{\epsilon_{i}=q_{k, j}^{*}-e_{i}} f\left(q_{k, j}^{*}-e_{i}\right)-\left.\frac{\partial y_{j}\left(e, e_{i}, \epsilon_{i}\right)}{\partial e_{i}}\right|_{\epsilon_{i}=q_{k, j+1}^{*}-e_{i}} f\left(q_{k, j+1}^{*}-e_{i}\right) \\
\left.+\int_{q_{k, j}^{*}-e_{i}}^{q_{k+1, j}^{*}-e_{i}} \frac{\partial^{2} y_{j}\left(e, e_{i}, \epsilon_{i}\right)}{\partial e_{i}^{2}} f\left(\epsilon_{i}\right) d \epsilon_{i}\right]-c^{\prime \prime}\left(e_{i}\right),
\end{array}
$$

where $\frac{\partial\left[y_{j}\left(e, e_{i}, q_{k, j}^{*}-e_{i}\right)\right]}{\partial e_{i}}$ denotes the partial derivative of $y_{j}\left(e, e_{i}, q_{k, j}^{*}-e_{i}\right)$ with respect to $e_{i}$, and $\left.\frac{\partial y_{j}\left(e, e_{i}, \epsilon_{i}\right)}{\partial e_{i}}\right|_{\epsilon_{i}=q_{k, j}^{*}-e_{i}}$ denotes the partial derivative of $y_{j}\left(e, e_{i}, \epsilon_{i}\right)$ with respect to $e_{i}$ evaluated at $\epsilon_{i}=q_{k, j}^{*}-e_{i}$. But $y_{j}\left(e, e_{i}, \epsilon_{i}\right)$ is just the probability that an agent finishes in $j$ th place for given values of $e, e_{i}$, and $\epsilon_{i}$ and is therefore a continuous and bounded function with bounded first and second derivatives (for fixed and finite $n$ ). Similarly, $f(\cdot)$ is just a continuous and bounded density with bounded first derivative. From this it follows that there exists some bound $C$ independent of $e, e_{i}$, and all the functions $g_{j}\left(q_{j}\right)$ such that if $c^{\prime \prime}(e)>C$ for all $e$, then the second derivative of the agent's utility with respect to effort is always negative. This implies that if the first-order conditions for a given level of effort $e_{i}$ to be a local optimum are satisfied, then this level of effort $e_{i}$ is also a global optimum. From this it follows that if $c(\cdot)$ is a sufficiently convex function, then any level of effort $e$ that satisfies

$$
\begin{array}{r}
\sum_{j=1}^{n} \sum_{k=0}^{m_{j}} v\left(r_{k, j} A\right)\left[\left(y_{j}\left(e, e, q_{k, j}^{*}-e\right) f\left(q_{k, j}^{*}-e\right)-y_{j}\left(e, e, q_{k+1, j}^{*}-e\right) f\left(q_{k+1, j}^{*}-e\right)\right)\right. \\
\left.+\left.\int_{q_{k, j}^{*}-e}^{q_{k+1, j}^{*}-e} \frac{\partial y_{j}\left(e, e_{i}, \epsilon_{i}\right)}{\partial e_{i}}\right|_{e_{i}=e} f\left(\epsilon_{i}\right) d \epsilon_{i}\right]-c^{\prime}(e)=0
\end{array}
$$

can indeed be sustained in a symmetric pure-strategy equilibrium.

To prove that there exists a unique symmetric pure-strategy equilibrium, it thus suffices to show that there is a unique value of $e$ that satisfies Equation (4). To see that such a value exists, note that when $e=0$, then $c^{\prime}(e)=0$, but the derivative of a player's expected prize with respect to effort is positive, so the derivative in Equation (4) is positive. But in the limit as $e \rightarrow \infty, c^{\prime}(e) \rightarrow \infty$, but the derivative of a player's expected prize with respect to effort must remain bounded, so Equation (4) becomes negative. Since the expression in Equation (4) is a continuous function of $e$, it then follows that there is some intermediate value of $e$ for which this equation is satisfied with equality.

To see that this equilibrium is unique for sufficiently convex $c(\cdot)$, note that similar reasoning to that used to show the second derivative in Equation (3) is negative also shows that the derivative of Equation (4) with respect to $e$ is negative for sufficiently convex $c(\cdot)$. This in turn implies that there is at most one solution to this equation. From this it follows that if $c(\cdot)$ is sufficiently convex, then there exists a unique symmetric pure-strategy equilibrium.

In addition to Theorem A.1, we also seek to show that the value of $C$ in the statement of this theorem that is needed to ensure existence of a unique symmetric pure-strategy equilibrium is 
minimal when the noise terms are drawn from a distribution with sufficiently high variance. First, we present a preliminary technical lemma about one of the terms in Equation (3) in the above proof:

Lemma A.2. The probability that agent $i$ will finish in $j$ th place for a given realization of $\epsilon_{i}$ when agent $i$ exerts effort $e_{i}$ and all other agents exert effort $e$ is

$$
y_{j}\left(e, e_{i}, \epsilon_{i}\right)=\left(\begin{array}{c}
n-1 \\
j-1
\end{array}\right)\left(1-F\left(e_{i}-e+\epsilon_{i}\right)\right)^{j-1} F\left(e_{i}-e+\epsilon_{i}\right)^{n-j} .
$$

Proof. If $\epsilon_{[j]}$ denotes the $j$ th-largest of the noise terms $\epsilon$ drawn by the $n-1$ remaining agents, then agent $i$ will have the $j$ th-highest quality output if and only if $e+\epsilon_{[j]} \leq e_{i}+\epsilon_{i} \leq e+\epsilon_{[j-1]}$. The probability of this event, for a given draw of $\epsilon_{i}$, is the probability that the number $e_{i}+\epsilon_{i}-e$ lies between the random values of $\epsilon_{[j]}$ and $\epsilon_{[j-1]}$. Thus, the probability of this event is equal to the probability that exactly $j-1$ of $n-1$ randomly drawn values from the cumulative distribution function $F(\cdot)$ exceed $e_{i}+\epsilon_{i}-e$. Using standard expressions for binomial probabilities, we know that this event takes place with the probability given in Equation (5).

With Lemma A.2 in mind, we now show that the value of $C$ that is needed in Theorem A.1 to ensure existence of a unique symmetric pure-strategy equilibrium is minimal when the variance in the noise terms is sufficiently large. In particular, we parameterize $F(\cdot)$ by $F_{\sigma}(\epsilon) \equiv F\left(\frac{\epsilon}{\sigma}\right)$ and consider what happens in the limit as $\sigma \rightarrow \infty$. In this setting, we obtain the following result:

Theorem A.3. If $F_{\sigma}(\epsilon) \equiv F\left(\frac{\epsilon}{\sigma}\right)$, then the minimum value of $c^{\prime \prime}(\cdot)$ needed to ensure existence of a unique symmetric pure-strategy equilibrium in Theorem A.1, $C=C\left(n, A_{j}, F_{\sigma}\right)$, approaches 0 in the limit as $\sigma \rightarrow \infty$.

Proof. If $f_{\sigma}(\epsilon)$ denotes the probability density function corresponding to $F_{\sigma}(\epsilon)$, then $f_{\sigma}(\epsilon)=$ $\frac{d}{d \epsilon} F\left(\frac{\epsilon}{\sigma}\right)=\frac{1}{\sigma} f\left(\frac{\epsilon}{\sigma}\right)$ and $f_{\sigma}^{\prime}(\epsilon)=\frac{1}{\sigma^{2}} f^{\prime}\left(\frac{\epsilon}{\sigma}\right)$. Thus, both $f_{\sigma}(\epsilon)$ and $f_{\sigma}^{\prime}(\epsilon)$ converge to 0 uniformly in the limit as $\sigma \rightarrow \infty$.

In addition to this, if $h(x) \equiv\left(\begin{array}{c}n-1 \\ j-1\end{array}\right)(1-x)^{j-1} x^{n-j}$, then we know from Equation (5) that $y_{j}\left(e, e_{i}, \epsilon_{i}\right)=\left(\begin{array}{c}n-1 \\ j-1\end{array}\right)\left(1-F_{\sigma}\left(e_{i}-e+\epsilon_{i}\right)\right)^{j-1} F_{\sigma}\left(e_{i}-e+\epsilon_{i}\right)^{n-j}=h\left(F_{\sigma}\left(e_{i}-e+\epsilon_{i}\right)\right)$. It then follows that $\frac{\partial y_{j}\left(e, e_{i}, \epsilon_{i}\right)}{\partial e_{i}}=h^{\prime}\left(F_{\sigma}\left(e_{i}-e+\epsilon_{i}\right)\right) f_{\sigma}\left(e_{i}-e+\epsilon_{i}\right)$ and $\frac{\partial^{2} y_{j}\left(e, e_{i}, \epsilon_{i}\right)}{\partial e_{i}^{2}}=h^{\prime \prime}\left(F_{\sigma}\left(e_{i}-e+\epsilon_{i}\right)\right) f_{\sigma}^{2}\left(e_{i}-\right.$ $\left.e+\epsilon_{i}\right)+h^{\prime}\left(F_{\sigma}\left(e_{i}-e+\epsilon_{i}\right)\right) f_{\sigma}^{\prime}\left(e_{i}-e+\epsilon_{i}\right)$. Thus, $\frac{\partial y_{j}\left(e, e_{i}, \epsilon_{i}\right)}{\partial e_{i}}$ and $\frac{\partial^{2} y_{j}\left(e, e_{i}, \epsilon_{i}\right)}{\partial e_{i}^{2}}$ both converge to 0 uniformly in the limit as $\sigma \rightarrow \infty$ as well.

But these results in turn imply that the expression in brackets in Equation (3) also converges to 0 uniformly in the limit as $\sigma \rightarrow \infty$. Thus, in the limit as $\sigma \rightarrow \infty$, the minimum value of $C$ needed to ensure that the expression in Equation (3) will be negative if $c^{\prime \prime}(e)>C$ approaches 0 , which in turn implies that the minimum value of $c^{\prime \prime}(\cdot)$ needed to ensure existence of a symmetric pure-strategy equilibrium approaches 0 in the limit as $\sigma \rightarrow \infty$. A similar argument shows that the minimum value of $c^{\prime \prime}(\cdot)$ needed to ensure any such equilibrium is unique approaches 0 in the limit as $\sigma \rightarrow \infty$.

\section{A.2 Optimality of Threshold Mechanisms}

Proof of Theorem 3.1: As in the proof of Theorem A.1, to prove that threshold mechanisms are optimal amongst all mechanisms, it suffices to show that threshold mechanisms are also optimal amongst the set of all mechanisms induced by ladder functions. We also know from this proof that 
in a pure-strategy equilibrium, the equilibrium effort $e$ must satisfy

$$
\begin{array}{r}
c^{\prime}(e)=\sum_{j=1}^{n} \sum_{k=0}^{m_{j}} v\left(r_{k, j} A\right)\left[\left(y_{j}\left(e, e, q_{k, j}^{*}-e\right) f\left(q_{k, j}^{*}-e\right)-y_{j}\left(e, e, q_{k+1, j}^{*}-e\right) f\left(q_{k+1, j}^{*}-e\right)\right)\right. \\
\left.+\left.\int_{q_{k, j}^{*}-e}^{q_{k+1, j}^{*}-e} \frac{\partial y_{j}\left(e, e_{i}, \epsilon_{i}\right)}{\partial e_{i}}\right|_{e_{i}=e} f\left(\epsilon_{i}\right) d \epsilon_{i}\right] .
\end{array}
$$

But note that the right-hand side of this equation is a linear function of $v\left(r_{k, j} A\right)$ for all $k$ and $j$. From this it follows that for all $k \leq m_{j}$, the right-hand side of this equation is either non-decreasing in $r_{k, j}$ or non-increasing in $r_{k, j}$. Thus, if $m_{j} \geq 2$, then one can instead set the value of $r_{1, j}$ to either be equal to $r_{0, j}$ or $r_{2, j}$ without decreasing the right-hand side of Equation (6), meaning that one can make this change without decreasing equilibrium effort.

But setting the value of $r_{1, j}$ to equal $r_{0, j}$ or $r_{2, j}$ would be equivalent to replacing the ladder function $l_{j}(q)$ with a ladder function that has $m_{j}-1$ points of discontinuity rather than $m_{j}$ points of discontinuity. From this it follows that if one is using a mechanism based on ladder functions $l_{j}(q)$ such that some $l_{j}(q)$ has $m_{j} \geq 2$ points of discontinuity, then the mechanism designer can induce at least as large a level of effort by instead using some mechanism based on ladder functions such that $l_{j}(q)$ has $m_{j}-1$ points of discontinuity. By induction, it follows that the mechanism designer can also induce at least as much effort by instead using some mechanism based on ladder functions such that each $l_{j}(q)$ has no more than one point of discontinuity.

To complete the proof, it suffices to show that if the mechanism designer is using a mechanism based on ladder functions $l_{j}(q)$ that each have no more than one point of discontinuity, then these single-step ladder functions must correspond to threshold mechanisms, i.e., that $r_{0, j} \in\{0,1\}$ and $r_{1, j} \in\{0,1\}$. Since equilibrium effort is given by the solution to Equation (6), and the right-hand side of this equation is a linear function of $v\left(r_{k, j} A\right)$ for all $k$ and $j$, we again have that the righthand side of this equation is either non-decreasing in $r_{0, j}$ or non-increasing in $r_{0, j}$. Thus, if $l_{j}(q)$ has exactly one point of discontinuity, then one can set the value of $r_{0, j}$ to either be equal to 0 or $r_{1, j}$ without decreasing the right-hand side of Equation (6), meaning that one can make this change without decreasing equilibrium effort.

Now, if $r_{0, j}=0$, then the same argument illustrates that one can set $r_{1, j}$ equal to 0 or 1 without decreasing the right-hand side of Equation (6), meaning one can make this change without decreasing equilibrium effort. And if $r_{0, j}=r_{1, j}$, then $l_{j}(q)$ has no points of discontinuity, and the same argument again illustrates that one can set the value of $r_{0, j}=r_{1, j}$ to be either 0 or 1 without decreasing the right-hand side of Equation (6), meaning that one can make this change without decreasing equilibrium effort. Thus, the mechanism designer can induce the agents to exert at least as much effort by using a threshold mechanism in which $r_{0, j} \in\{0,1\}$ and $r_{1, j} \in\{0,1\}$. The result follows.

\section{A.3 Optimal Thresholds}

Lemma A.4. Consider the threshold mechanism $\mathcal{M}\left(A_{1}, t_{1}, A_{2}, t_{2}, \ldots, A_{n}, t_{n}\right)$, where the $j$ th-ranked agent receives prize $A_{j}$ if and only if her output quality exceeds $t_{j}$. Under this mechanism, a player's expected utility from exerting effort $e_{i}$ if all other players exert effort $e$ is

$$
E\left[u_{i}\right]=\sum_{j=1}^{k} v\left(A_{j}\right) \int_{t_{j}-e_{i}}^{\infty}\left(\begin{array}{c}
n-1 \\
j-1
\end{array}\right)\left(1-F\left(e_{i}-e+\epsilon_{i}\right)\right)^{j-1} F\left(e_{i}-e+\epsilon_{i}\right)^{n-j} f\left(\epsilon_{i}\right) d \epsilon_{i}-c\left(e_{i}\right) .
$$

Proof. The probability that agent $i$ will finish in $j$ th place for a given realization of $\epsilon_{i}$ when agent $i$ exerts effort $e_{i}$ and all other agents exert effort $e$ is given by the expression in Equation (5). Now suppose agent $i$ produces output $q_{i}=e_{i}+\epsilon_{i}$. To actually receive the prize $A_{j}$ in the threshold 
mechanism $\mathcal{M}\left(A_{1}, t_{1}, A_{2}, t_{2}, \ldots, A_{n}, t_{n}\right)$, $i$ 's output must also exceed the threshold $t_{j}$, i.e., $e_{i}+\epsilon_{i} \geq$ $t_{j}$. So the probability that agent $i$ finally receives the $j$ th prize, unconditional on the realization of $\epsilon_{i}$, is the integral of the probability in Equation (5) over $\epsilon_{i} \geq t_{j}-e_{i}$, i.e.,

$$
\int_{t_{j}-e_{i}}^{\infty}\left(\begin{array}{c}
n-1 \\
j-1
\end{array}\right)\left(1-F\left(e_{i}-e+\epsilon_{i}\right)\right)^{j-1} F\left(e_{i}-e+\epsilon_{i}\right)^{n-j} f\left(\epsilon_{i}\right) d \epsilon_{i} .
$$

Thus, an agent's expected utility from exerting effort $e_{i}$, when other agents exert effort $e$, is the sum of her expected benefit over all ranks $j$ minus her cost or

$$
E\left[u_{i}\right]=\sum_{j=1}^{k} v\left(A_{j}\right) \int_{t_{j}-e_{i}}^{\infty}\left(\begin{array}{c}
n-1 \\
j-1
\end{array}\right)\left(1-F\left(e_{i}-e+\epsilon_{i}\right)\right)^{j-1} F\left(e_{i}-e+\epsilon_{i}\right)^{n-j} f\left(\epsilon_{i}\right) d \epsilon_{i}-c\left(e_{i}\right) .
$$

Proof of Theorem 4.1: If the mechanism designer uses a threshold mechanism in which the agent who finishes in $j$ th place receives a prize if and only if this agent's observed quality exceeds $t_{j}$, then an agent $i$ 's expected utility from exerting effort $e_{i}$ when all other agents are exerting effort $e$ is given by

$$
E\left[u_{i}\right]=\sum_{j=1}^{n} \int_{t_{j}-e_{i}}^{\infty} v\left(A_{j}\right) y_{j}\left(e, e_{i}, \epsilon_{i}\right) f\left(\epsilon_{i}\right) d \epsilon_{i}-c\left(e_{i}\right),
$$

where we let $y_{j}\left(e, e_{i}, \epsilon_{i}\right) \equiv\left(\begin{array}{c}n-1 \\ j-1\end{array}\right)\left(1-F\left(e_{i}-e+\epsilon_{i}\right)\right)^{j-1} F\left(e_{i}-e+\epsilon_{i}\right)^{n-j}$ denote the probability that agent $i$ finishes in $j$ th place given that all other agents exert effort $e$, agent $i$ exerts effort $e_{i}$, and the value of agent $i$ 's noise term is $\epsilon_{i}$. From this it follows that the derivative of the agent's utility with respect to $e_{i}$ is given by the following expression:

$$
\sum_{j=1}^{n} v\left(A_{j}\right)\left[y_{j}\left(e, e_{i}, t_{j}-e_{i}\right) f\left(t_{j}-e_{i}\right)+\int_{t_{j}-e_{i}}^{\infty} \frac{\partial y_{j}\left(e, e_{i}, \epsilon_{i}\right)}{\partial e_{i}} f\left(\epsilon_{i}\right) d \epsilon_{i}\right]-c^{\prime}\left(e_{i}\right) .
$$

By setting this derivative equal to zero when $e_{i}=e$, it then follows that it is an equilibrium for all agents to exert effort $e$ if and only if

$$
\sum_{j=1}^{n} v\left(A_{j}\right)\left[y_{j}\left(e, e, t_{j}-e\right) f\left(t_{j}-e\right)+\left.\int_{t_{j}-e}^{\infty} \frac{\partial y_{j}\left(e, e_{i}, \epsilon_{i}\right)}{\partial e_{i}}\right|_{e_{i}=e} f\left(\epsilon_{i}\right) d \epsilon_{i}\right]=c^{\prime}(e) .
$$

In the optimal threshold mechanism, the thresholds $t_{j}$ must be chosen in such a way as to make the expression in Equation (7) as large as possible for a given $e$. A necessary condition for this is that the derivative of the left-hand side of this equation with respect to $t_{j}$ must be zero for all $j$. Differentiating the left-hand side of this equation with respect to $t_{j}$ gives

$$
\begin{aligned}
v\left(A_{j}\right) y_{j}\left(e, e, t_{j}-e\right) f^{\prime}\left(t_{j}-e\right) & +v\left(A_{j}\right)\left[\frac{\partial\left[y_{j}\left(e, e, t_{j}-e\right)\right]}{\partial t_{j}}-\left.\frac{\partial y_{j}\left(e, e_{i}, \epsilon_{i}\right)}{\partial e_{i}}\right|_{e_{i}=e, \epsilon_{i}=t_{j}-e}\right] f\left(t_{j}-e\right) \\
& =v\left(A_{j}\right) y_{j}\left(e, e, t_{j}-e\right) f^{\prime}\left(t_{j}-e\right),
\end{aligned}
$$

where the equality follows from the fact that the partial derivatives of $y_{j}\left(e, e_{i}, \epsilon_{i}\right)=\left(\begin{array}{c}n-1 \\ j-1\end{array}\right)(1-$ $\left.F\left(e_{i}-e+\epsilon_{i}\right)\right)^{j-1} F\left(e_{i}-e+\epsilon_{i}\right)^{n-j}$ with respect to $e_{i}$ and $\epsilon_{i}$ are equal.

Now note that the principal would never have an incentive to choose a value of $t_{j}$ equal to $-\infty$ or $\infty$. To see this, note that for values of $t_{j}$ that are arbitrarily negative, it must be the case that $t_{j}-e<$ 0 in equilibrium, meaning $f^{\prime}\left(t_{j}-e\right)>0$ and the derivative of the left-hand side of Equation (7) with respect to $t_{j}$ is positive. Thus, for any sufficiently negative values of $t_{j}$, the principal can always increase the left-hand side of Equation (7) by increasing $t_{j}$, and thereby increase the equilibrium level of effort. From this it follows that a threshold of $t_{j}=-\infty$ can never be optimal for any $j$. A 
similar argument shows that a threshold of $t_{j}=\infty$ can also never be optimal. Thus, there is some large finite value of $T>0$ such that all thresholds $t_{j} \notin[-T, T]$ are dominated by some threshold $t_{j} \in[-T, T]$ for all $j$. Since this set of feasible thresholds is compact, and the equilibrium level of effort varies continuously with the thresholds, it then follows that some set of optimal thresholds exists.

Now if the optimal threshold $t_{j}$ is not equal to $-\infty$ or $\infty$, then the derivative in Equation (8) must be zero at the optimal $t_{j}$. Now, we know that $f(\cdot)$ is single-peaked at 0 , so when $t_{j}$ is not equal to $-\infty$ or $\infty$, the above derivative is only zero when $t_{j}=e$. Thus, the optimal thresholds satisfy $t_{j}=e$ for all $j$ and the optimal thresholds are always equal for all prizes.

Proof of Theorem 4.3: Note that a player $i$ 's expected value for her prize from exerting effort $e_{i}$ when all other players are exerting effort $e$ is just equal to the sum, over all $j=1, \ldots, n$, of the difference between the value the player obtains from receiving the $j$ th prize $A_{j}$ and her value from the $j+1$ th prize $A_{j+1}$, multiplied by the probability that she finishes in at least $j$ th place and meets the threshold $t$. Now let $G_{j}(q)$ denote the probability that no more than $j-1$ of the $n-1$ values of $\epsilon_{k}$ are greater than $q$. Then the probability that agent $i$ finishes in at least $j$ th place for any given realization of $\epsilon_{i}$ when she exerts effort $e_{i}$ and all other players are exerting effort $e$ is $G_{j}\left(e_{i}-e+\epsilon_{i}\right)$. From this it follows that the probability that agent $i$ finishes in at least $j$ th place and meets the threshold $t$ unconditional on the realization of $\epsilon_{i}$ is

$$
\int_{t-e_{i}}^{\infty} G_{j}\left(e_{i}-e+\epsilon_{i}\right) f\left(\epsilon_{i}\right) d \epsilon_{i}
$$

From the previous paragraph, it follows that player $i$ 's expected utility from exerting effort $e_{i}$ when all other players are exerting effort $e$ is

$$
\sum_{j=1}^{n}\left(v\left(A_{j}\right)-v\left(A_{j+1}\right)\right) \int_{t-e_{i}}^{\infty} G_{j}\left(e_{i}-e+\epsilon_{i}\right) f\left(\epsilon_{i}\right) d \epsilon_{i}-c\left(e_{i}\right) .
$$

By differentiating this expression with respect to $e_{i}$, setting the derivative equal to zero, and using the fact that $e_{i}=e$ must hold in any symmetric pure-strategy equilibrium, it follows that the following relationship must be satisfied by the equilibrium level of $e$ :

$$
\sum_{j=1}^{n}\left(v\left(A_{j}\right)-v\left(A_{j+1}\right)\right)\left[G_{j}(t-e) f(t-e)+\int_{t-e}^{\infty} \frac{d G_{j}(\epsilon)}{d \epsilon} f(\epsilon) d \epsilon\right]=c^{\prime}(e) .
$$

Now the derivative of the left-hand side of Equation (9) with respect to $t-e$ is

$$
\sum_{j=1}^{n}\left(v\left(A_{j}\right)-v\left(A_{j+1}\right)\right) G_{j}(t-e) f^{\prime}(t-e),
$$

which is positive when $t-e<0$. Note that if $t<t^{*}$, where $t^{*}$ denotes the optimal threshold, then the equilibrium effort corresponding to that threshold, $e^{*}(t)$, must satisfy $t<e^{*}(t)<t^{*}$ for the following reason: If we had $e=t$, then the value of the left-hand side of Equation (9) would be the same at $t$ as it is at $t^{*}$ but the value of the right-hand side would be strictly lower. Similarly, if we had $e=t^{*}$, then the right-hand side of Equation (9) would be the same at $t$ as it is at $t^{*}$, but the left-hand side would be strictly lower at $t$ than it is at $t^{*}$. By combining these facts, the continuity of $f(\cdot), f^{\prime}(\cdot)$, and $G(\cdot)$, and the intermediate value theorem, it follows that there must exist some $e^{*}(t)$ satisfying $t<e^{*}(t)<t^{*}$ such that the left and right-hand sides of Equation (9) are equal. Thus, equilibrium effort satisfies $t<e^{*}(t)<t^{*}$.

Next, we seek to show that equilibrium effort is increasing in $t$ when $t<t^{*}$. To prove this, consider two thresholds $t_{1}$ and $t_{2}$ such that $t_{1}<t_{2}<t^{*}$. It suffices to show that $e\left(t_{1}\right)<e\left(t_{2}\right)$. To see 
that this holds, note that it must be the case that Equation (9) is satisfied with equality when $t=t_{2}$ and $e=e\left(t_{2}\right)$. Now if $e\left(t_{1}\right) \geq e\left(t_{2}\right)$, then it must be the case that the left-hand side of Equation (9) is lower when $t=t_{1}$ and $e=e\left(t_{1}\right)$ than when $t=t_{2}$ and $e=e\left(t_{2}\right)$, since the derivative of the lefthand side of this equation with respect to $t-e$ is positive when $t-e<0$. And it must also be the case that the right-hand side of Equation (9) is no smaller when $e=e\left(t_{1}\right)$ than when $e=e\left(t_{2}\right)$, since the right-hand side of this equation is increasing in $e$. But this would imply that the left-hand side of Equation (9) is lower than the right-hand side of this equation when $t=t_{1}$ and $e=e\left(t_{1}\right)$, contradicting the possibility that $e\left(t_{1}\right)$ is an equilibrium level of effort. Thus, $e\left(t_{1}\right) \geq e\left(t_{2}\right)$ cannot hold.

Next, we show that if $t>t^{*}$, then $e^{*}(t)<t^{*}<t$. To see this, note that the definition of $t^{*}$ is that $t^{*}$ is the threshold that results in the highest equilibrium effort. Thus, $e^{*}(t)<e^{*}\left(t^{*}\right)$ for any $t \neq t^{*}$. Since $e^{*}\left(t^{*}\right)=t^{*}$ from Corollary 4.2, it then follows that $e^{*}(t)<t^{*}<t$.

Finally, we show that if $t>t^{*}$, then equilibrium effort is decreasing in $t$. To see this, consider some thresholds $t_{1}$ and $t_{2}$ satisfying $t^{*}<t_{1}<t_{2}$. Note that when $t=t_{1}$ and $e=e\left(t_{1}\right)$, the equilibrium condition Equation (9) is satisfied. Now $t_{1}>e\left(t_{1}\right)$, so $t_{1}-e\left(t_{1}\right)>0$, and we know that the derivative of the left-hand side of Equation (9) with respect to $t$ is negative. Thus, when $t=t_{2}$ and $e=e\left(t_{1}\right)$, the left-hand side of Equation (9) is less than the right-hand side of Equation (9). But when $t=t_{2}$ and $e=0$, then the left-hand side of Equation (9) is greater than the right-hand side of Equation $(9)\left(\right.$ since $c^{\prime}(0)=0$ ). By the intermediate value theorem, it then follows that there exists some $e\left(t_{2}\right) \in\left(0, e\left(t_{1}\right)\right)$ such that Equation (9) is satisfied with equality when $t=t_{2}$ and $e=e\left(t_{2}\right)$. Thus, $t^{*}<t_{1}<t_{2}$ implies $e\left(t_{2}\right)<e\left(t_{1}\right)$, meaning that if $t>t^{*}$, then equilibrium effort is decreasing in $t$.

Proof of Theorem 4.4: Recall from Equation (9) applied to the case where there are $k$ prizes that if $G_{j}(q)$ denotes the probability that no more than $j-1$ of the $n-1$ values of $\epsilon_{k}$ are greater than $q$, then the following equality must hold for the equilibrium effort $e$ when the principal uses the threshold $t$ :

$$
\sum_{j=1}^{k}\left(v\left(A_{j}\right)-v\left(A_{j+1}\right)\right)\left[G_{j}(t-e) f(t-e)+\int_{t-e}^{\infty} \frac{d G_{j}(\epsilon)}{d \epsilon} f(\epsilon) d \epsilon\right]=c^{\prime}(e) .
$$

From Corollary 4.2, the equilibrium effort $e(t)$ at the optimal threshold $t^{*}$ satisfies $t^{*}=e\left(t^{*}\right)$, so $t-e=0$ in the equation above. Substituting, we have that equilibrium effort in the optimal threshold mechanism is the solution to

$$
\sum_{j=1}^{k}\left(v\left(A_{j}\right)-v\left(A_{j+1}\right)\right)\left[G_{j}(0) f(0)+\int_{0}^{\infty} \frac{d G_{j}(\epsilon)}{d \epsilon} f(\epsilon) d \epsilon\right]=c^{\prime}(e) .
$$

Here, $\frac{d G_{j}(\epsilon)}{d \epsilon}$ represents the density corresponding to the distribution $G(\epsilon)$. Write $G_{j}(\epsilon ; n)$ to denote the dependence of $G_{j}$ on $n$. Then, the distribution $G_{j}\left(\epsilon ; n^{\prime}\right)$ first-order stochastically dominates $G_{j}(\epsilon ; n)$ for all $n^{\prime}>n$. Further, $f(\epsilon)$ is non-increasing in $\epsilon$ for all $\epsilon \geq 0$, since $f(\cdot)$ is single-peaked at 0 . From this it follows that increasing $n$ decreases the value of

$$
G_{j}(0) f(0)+\int_{0}^{\infty} \frac{d G_{j}(\epsilon)}{d \epsilon} f(\epsilon) d \epsilon=E_{\epsilon \sim G_{j}}[f(\max \{0, \epsilon\})]
$$

for all $j$. Therefore, for equality to hold in Equation (11), the equilibrium effort $e(n)$ must be such that $c^{\prime}(e(n))$ also decreases with $n$, i.e., $e(n)$ decreases with $n$. Since the equilibrium effort in the optimal threshold mechanism decreases with the number of players, and the optimal threshold equals equilibrium effort in the optimal threshold mechanism, by Corollary 4.2, the optimal threshold $t^{*}(n)$ is also decreasing in $n$. 
Proof of Theorem 4.5: We know from Equation (11) in the proof of the previous theorem that if $G_{j}(q)$ denotes the probability that no more than $j-1$ of the $n-1$ other values of $\epsilon_{k}$ are greater than $q$, then the following condition must be satisfied by the equilibrium level of effort $e$ in any pure-strategy equilibrium of the optimal threshold mechanism:

$$
\sum_{j=1}^{k}\left(v\left(A_{j}\right)-v\left(A_{j+1}\right)\right)\left[G_{j}(0) f(0)+\int_{0}^{\infty} \frac{d G_{j}(\epsilon)}{d \epsilon} f(\epsilon) d \epsilon\right]=c^{\prime}(e) .
$$

Substituting in the fact that $A_{j}=A_{k}$ for $j \leq k$ and $A_{j}=0$ for $j>k$, it then follows that the equilibrium level of effort $e$ satisfies the following equation in any pure-strategy equilibrium:

$$
v\left(A_{k}\right)\left[G_{k}(0) f(0)+\int_{0}^{\infty} \frac{d G_{k}(\epsilon)}{d \epsilon} f(\epsilon) d \epsilon\right]=c^{\prime}(e),
$$

which can be rewritten as

$$
v\left(A_{k}\right) E_{\epsilon \sim G_{k}}[f(\max \{0, \epsilon\})]=c^{\prime}(e) .
$$

Now if one increases the number of prizes by awarding additional prizes that are the same as those originally awarded to agents who finished in the top $k$ and met the threshold, then $v\left(A_{k}\right)$ is independent of $k$. And if one increases the number of prizes by splitting the same total prize pool amongst a larger number of players, then in the limit as the minimum coefficient of absolute risk aversion, $\alpha \equiv \inf _{A}-\frac{v^{\prime \prime}(A)}{v^{\prime}(A)}$, becomes arbitrarily large, $\frac{v\left(A_{k}\right)}{v(A)}$ becomes arbitrarily close to 1 , and $v\left(A_{k}\right)$ approaches a function that is also independent of $k$.

We also know that $E_{\epsilon \sim G_{k}}[f(\max \{0, \epsilon\})]$ is increasing in $k$ for the following reason: The fact that $G_{k}(\epsilon)$ is a distribution corresponding to the probability that no more than $k-1$ of the $n-1$ values of $\epsilon_{j}$ are greater than $\epsilon$ implies that $G_{j}$ first-order stochastically dominates $G_{k}$ for all $j<k$. And we also know that $f(\max \{0, \epsilon\})$ is decreasing in $\epsilon$ for all $\epsilon>0$. By combining these facts, it follows that $E_{\epsilon \sim G_{k}}[f(\max \{0, \epsilon\})]$ is increasing in $k$.

Thus, under either of the conditions of the theorem, it must be the case that $v\left(A_{k}\right)$ $E_{\epsilon \sim G_{k}}[f(\max \{0, \epsilon\})]$ is increasing in $k$. By combining this fact with Equation (12), it then follows that under either of these conditions, equilibrium effort in the optimal threshold mechanism is increasing in $k$. Since the optimal threshold is equal to equilibrium effort in the optimal threshold mechanism, it then follows that the optimal threshold is also increasing in $k$ under the conditions of the theorem.

\section{A.4 How Useful is Cardinal Information?}

Proof of Theorem 5.1: (1) Recall from Equation (10) that if $G_{j}(q)$ denotes the probability that no more than $j-1$ of the $n-1$ values of $\epsilon_{k}$ are greater than $q$, then equilibrium effort $e$ is pinned down by the following equation:

$$
\sum_{j=1}^{k}\left(v\left(A_{j}\right)-v\left(A_{j+1}\right)\right)\left[G_{j}(t-e) f(t-e)+\int_{t-e}^{\infty} \frac{d G_{j}}{d \epsilon}(\epsilon) f(\epsilon) d \epsilon\right]=c^{\prime}(e) .
$$

Now, if $c(e)=\frac{e^{2}}{2}$, then $c^{\prime}(e)=e$. We also know that under the optimal threshold, $t=e$ in equilibrium, so the equilibrium effort $e$ is given by

$$
e_{o p t}=\sum_{j=1}^{k}\left(v\left(A_{j}\right)-v\left(A_{j+1}\right)\right)\left[G_{j}(0) f(0)+\int_{0}^{\infty} \frac{d G_{j}}{d \epsilon}(\epsilon) f(\epsilon) d \epsilon\right] .
$$


When there is no threshold, we have $t-e=-\infty$, so

$$
e_{0}=\sum_{j=1}^{k}\left(v\left(A_{j}\right)-v\left(A_{j+1}\right)\right)\left[\int_{-\infty}^{\infty} \frac{d G_{j}}{d \epsilon}(\epsilon) f(\epsilon) d \epsilon\right] .
$$

By combining the results in the two previous equations, we see that

$$
\begin{aligned}
e_{o p t}-e_{0} & =\sum_{j=1}^{k}\left(v\left(A_{j}\right)-v\left(A_{j+1}\right)\right)\left[G_{j}(0) f(0)-\int_{-\infty}^{0} \frac{d G_{j}}{d \epsilon}(\epsilon) f(\epsilon) d \epsilon\right] \\
& =\sum_{j=1}^{k}\left(v\left(A_{j}\right)-v\left(A_{j+1}\right)\right)\left[\int_{-\infty}^{0} \frac{d G_{j}}{d \epsilon}(\epsilon)(f(0)-f(\epsilon)) d \epsilon\right] .
\end{aligned}
$$

Now, if $G_{j}(q)$ denotes the probability that no more than $j-1$ of the $n-1$ values of $\epsilon_{k}$ are greater than $q$, then we know that $\frac{d G_{j}}{d \epsilon}(\epsilon)=\frac{(n-1) !}{(j-1) !(n-j-1) !}(1-F(\epsilon))^{j-1} F(\epsilon)^{n-j-1} f(\epsilon)$. Thus, increasing $n$ by one changes the value of the integrand in this expression by a factor of $\frac{n}{n-j} F(\epsilon)<1$, where the inequality follows from the facts that $\frac{n}{n-j} \leq 2$ whenever there are at least twice as many players as the number of prizes and $F(\epsilon)<\frac{1}{2}$ for $\epsilon<0$. But this means that $e_{o p t}-e_{0}$ decreases when $n$ increases by one. The result then follows.

(2) Since the prizes are equal, $v\left(A_{j}\right)-v\left(A_{j+1}\right)=0$ for all $j \neq k$ and $v\left(A_{k}\right)-v\left(A_{k+1}\right)=v\left(A_{k}\right)$. We also know that $\frac{d G_{k}}{d \epsilon}(\epsilon)=\frac{(n-1) !}{(k-1) !(n-k-1) !}(1-F(\epsilon))^{k-1} F(\epsilon)^{n-k-1} f(\epsilon)$. From Equations (13) and (14), we then know that

$$
e_{0}=v\left(A_{k}\right)\left[\int_{-\infty}^{\infty} \frac{(n-1) !}{(k-1) !(n-k-1) !}(1-F(\epsilon))^{k-1} F(\epsilon)^{n-k-1} f^{2}(\epsilon) d \epsilon\right]
$$

and

$$
e_{o p t}-e_{0}=v\left(A_{k}\right)\left[\int_{-\infty}^{0} \frac{(n-1) !}{(k-1) !(n-k-1) !}(1-F(\epsilon))^{k-1} F(\epsilon)^{n-k-1} f(\epsilon)(f(0)-f(\epsilon)) d \epsilon\right] .
$$

Thus, we know that the ratio

$$
\begin{aligned}
\frac{e_{\text {opt }}-e_{0}}{e_{0}} & =\frac{\int_{-\infty}^{0}(1-F(\epsilon))^{k-1} F(\epsilon)^{n-k-1} f(\epsilon)(f(0)-f(\epsilon)) d \epsilon}{\int_{-\infty}^{\infty}(1-F(\epsilon))^{k-1} F(\epsilon)^{n-k-1} f^{2}(\epsilon) d \epsilon} \\
& =\frac{\int_{-\infty}^{0} H_{n, k}(\epsilon)(f(0)-f(\epsilon)) d \epsilon}{\int_{-\infty}^{0} H_{n, k}(\epsilon) f(\epsilon) d \epsilon+\int_{0}^{\infty} H_{n, k}(\epsilon) f(\epsilon) d \epsilon},
\end{aligned}
$$

where $H_{n, k}(\epsilon) \equiv(1-F(\epsilon))^{k-1} F(\epsilon)^{n-k-1} f(\epsilon)$. Increasing $n$ by one would then change the value of this ratio to

$$
\begin{aligned}
\frac{e_{o p t}-e_{0}}{e_{0}} & =\frac{\int_{-\infty}^{0}(1-F(\epsilon))^{k-1} F(\epsilon)^{n-k} f(\epsilon)(f(0)-f(\epsilon)) d \epsilon}{\int_{-\infty}^{\infty}(1-F(\epsilon))^{k-1} F(\epsilon)^{n-k} f^{2}(\epsilon) d \epsilon} \\
& =\frac{\int_{-\infty}^{0} H_{n, k}(\epsilon) F(\epsilon)(f(0)-f(\epsilon)) d \epsilon}{\int_{-\infty}^{0} H_{n, k}(\epsilon) F(\epsilon) f(\epsilon) d \epsilon+\int_{0}^{\infty} H_{n, k}(\epsilon) F(\epsilon) f(\epsilon) d \epsilon} .
\end{aligned}
$$


Now, $f(0)-f(\epsilon)$ is decreasing in $\epsilon$ for $\epsilon \leq 0$ and $f(\epsilon)$ and $F(\epsilon)$ are increasing in $\epsilon$ for $\epsilon \leq 0$. Thus,

$$
\frac{\int_{-\infty}^{0} H_{n, k}(\epsilon) F(\epsilon)(f(0)-f(\epsilon)) d \epsilon}{\int_{-\infty}^{0} H_{n, k}(\epsilon) F(\epsilon) f(\epsilon) d \epsilon}<\frac{\int_{-\infty}^{0} H_{n, k}(\epsilon)(f(0)-f(\epsilon)) d \epsilon}{\int_{-\infty}^{0} H_{n, k}(\epsilon) f(\epsilon) d \epsilon},
$$

since multiplying the terms in the $\int_{-\infty}^{0} H_{n, k}(\epsilon)(f(0)-f(\epsilon)) d \epsilon$ integral by $F(\epsilon)$ puts relatively more weight on the values of $\epsilon$ where the integrand is relatively smaller than multiplying the terms in the $\int_{-\infty}^{0} H_{n, k}(\epsilon) f(\epsilon) d \epsilon$ integral by $F(\epsilon)$. Further, note that

$$
\frac{\int_{-\infty}^{0} H_{n, k}(\epsilon) F(\epsilon) f(\epsilon) d \epsilon}{\int_{0}^{\infty} H_{n, k}(\epsilon) F(\epsilon) f(\epsilon) d \epsilon}<\frac{\int_{-\infty}^{0} H_{n, k}(\epsilon) f(\epsilon) d \epsilon}{\int_{0}^{\infty} H_{n, k}(\epsilon) f(\epsilon) d \epsilon},
$$

since $F(\epsilon)<(>) \frac{1}{2}$ for all $\epsilon<(>)$. Combining these results shows that

$$
\frac{\int_{-\infty}^{0} H_{n, k}(\epsilon) F(\epsilon)(f(0)-f(\epsilon)) d \epsilon}{\int_{-\infty}^{0} H_{n, k}(\epsilon) F(\epsilon) f(\epsilon) d \epsilon+\int_{0}^{\infty} H_{n, k}(\epsilon) F(\epsilon) f(\epsilon) d \epsilon}=\frac{a \int_{-\infty}^{0} H_{n, k}(\epsilon)(f(0)-f(\epsilon)) d \epsilon}{b \int_{-\infty}^{0} H_{n, k}(\epsilon) f(\epsilon) d \epsilon+c \int_{0}^{\infty} H_{n, k}(\epsilon) f(\epsilon) d \epsilon}
$$

for some positive constants $a, b$, and $c$ satisfying $a<b<c$. Thus,

$$
\frac{\int_{-\infty}^{0} H_{n, k}(\epsilon) F(\epsilon)(f(0)-f(\epsilon)) d \epsilon}{\int_{-\infty}^{0} H_{n, k}(\epsilon) F(\epsilon) f(\epsilon) d \epsilon+\int_{0}^{\infty} H_{n, k}(\epsilon) F(\epsilon) f(\epsilon) d \epsilon}<\frac{\int_{-\infty}^{0} H_{n, k}(\epsilon)(f(0)-f(\epsilon)) d \epsilon}{\int_{-\infty}^{0} H_{n, k}(\epsilon) f(\epsilon) d \epsilon+\int_{0}^{\infty} H_{n, k}(\epsilon) f(\epsilon) d \epsilon} .
$$

Thus, the value of the ratio $\frac{e_{\text {opt }}-e_{0}}{e_{0}}$ decreases when $n$ increases by one, which proves the result.

(3) We have seen in the proof of part (2) that

$$
e_{o p t}-e_{0}=v\left(A_{k}\right)\left[\int_{-\infty}^{0} \frac{(n-1) !}{(k-1) !(n-k-1) !}(1-F(\epsilon))^{k-1} F(\epsilon)^{n-k-1} f(\epsilon)(f(0)-f(\epsilon)) d \epsilon\right] .
$$

Increasing $k$ by one changes the value of the integrand in this expression by a factor of $\frac{n-k-1}{k} \frac{1-F(\epsilon)}{F(\epsilon)} \frac{v\left(A_{k+1}\right)}{v\left(A_{k}\right)}>\frac{n-k-1}{k} \frac{k}{k+1}=\frac{n-k-1}{k+1}$, where the inequality follows from the fact that $F(\epsilon)<\frac{1}{2}$ and $\frac{1-F(\epsilon)}{F(\epsilon)}>1$ for all $\epsilon<0$. Since $\frac{n-k-1}{k+1} \geq 1$ for $n \geq 2(k+1)$, it then follows that if the number of players is at least twice as large as the number of prizes, then the expression for $e_{o p t}-e_{0}$ increases when $k$ increases by one.

(4) The proof of this part is substantively identical to that in part (2) and thus omitted.

(5) Note that the values of $c^{\prime}\left(e_{o p t}\right)$ and $c^{\prime}\left(e_{0}\right)$ are independent of the cost function $c(\cdot)$, because under the optimal threshold, $t=e$ in equilibrium, so Equation (10) implies that equilibrium effort is given by the equation

$$
c^{\prime}\left(e_{o p t}\right)=\sum_{j=1}^{k}\left(v\left(A_{j}\right)-v\left(A_{j+1}\right)\right)\left[G_{j}(0) f(0)+\int_{0}^{\infty} \frac{d G_{j}}{d \epsilon}(\epsilon) f(\epsilon) d \epsilon\right],
$$

and when there is no threshold, we have $t-e=-\infty$, so

$$
c^{\prime}\left(e_{0}\right)=\sum_{j=1}^{k}\left(v\left(A_{j}\right)-v\left(A_{j+1}\right)\right)\left[\int_{-\infty}^{\infty} \frac{d G_{j}}{d \epsilon}(\epsilon) f(\epsilon) d \epsilon\right] .
$$

Thus, the values of $c^{\prime}\left(e_{o p t}\right)$ and $c^{\prime}\left(e_{0}\right)$ are the same for all $c(\cdot)$.

Now, suppose $\frac{c^{\prime \prime}(e)}{c^{\prime}(e)}=h(e)$ for some function $h(e)$. In that case, we know that $\ln \left(c^{\prime}\left(e_{o p t}\right)\right)-$ $\ln \left(c^{\prime}\left(e_{0}\right)\right)=\int_{e_{0}}^{e_{o p t}} h(e) d e$. Since $c^{\prime}\left(e_{o p t}\right)$ and $c^{\prime}\left(e_{0}\right)$ are the same for all $c(\cdot)$, this implies that for any cost function $c(\cdot), \int_{e_{0}}^{e_{o p t}} h(e) d e$ is equal to some constant independent of $c(\cdot)$. 
But if $c_{2}(\cdot)$ is more convex than $c_{1}(\cdot)$ in the sense that the ratio $\frac{c^{\prime \prime}(e)}{c^{\prime}(e)}$ is greater for $c=c_{2}(\cdot)$ than it is for $c=c_{1}(\cdot)$, then if $h_{2}(e) \equiv \frac{c_{2}^{\prime \prime}(e)}{c_{2}^{\prime}(e)}$ and $h_{1}(e) \equiv \frac{c_{1}^{\prime \prime}(e)}{c_{1}^{\prime}(e)}$, we have $h_{2}(e)>h_{1}(e)$ for all $e$. And if $e_{o p t, 1}$ and $e_{o p t, 2}$ denote the equilibrium levels of effort under the optimal threshold for the cost functions $c_{1}(\cdot)$ and $c_{2}(\cdot)$, then we know from the previous paragraph that $\int_{e_{0}}^{e_{o p t, 1}} h_{1}(e) d e=\int_{e_{0}}^{e_{o p t, 2}} h_{2}(e) d e$. But since $h_{2}(e)>h_{1}(e)$ for all $e$, it follows that for this equality to hold, we must have $e_{o p t, 1}>e_{o p t, 2}$. This in turn implies that both $e_{o p t}-e_{0}$ and the ratio $\frac{e_{o p t}-e_{0}}{e_{0}}$ are larger for $c_{1}(\cdot)$ than for $c_{2}(\cdot)$.

\section{A.5 Optimal Prize Structures}

Proof of Theorem 6.1: (1) We know from Equation (7) that the equilibrium level of effort is given by the solution to the equation

$$
\begin{aligned}
c^{\prime}(e)= & \sum_{j=1}^{n} v\left(A_{j}\right)\left[\left(\begin{array}{c}
n-1 \\
j-1
\end{array}\right)\left(1-F\left(t_{j}-e\right)\right)^{j-1} F\left(t_{j}-e\right)^{n-j} f\left(t_{j}-e\right)\right. \\
& +\int_{t_{j}-e}^{\infty}\left[\frac{(n-1) !}{(j-1) !(n-j-1) !}(1-F(\epsilon))^{j-1} F(\epsilon)^{n-j-1}\right. \\
& \left.\left.-\frac{(n-1) !}{(j-2) !(n-j) !}(1-F(\epsilon))^{j-2} F(\epsilon)^{n-j}\right] f^{2}(\epsilon) d \epsilon\right] .
\end{aligned}
$$

Under the optimal threshold mechanism, $t_{j}=e$ for all $j$ in equilibrium, so the equilibrium effort $e$ is given by

$$
\begin{aligned}
c^{\prime}(e)= & \sum_{j=1}^{n} v\left(A_{j}\right)\left[\left(\begin{array}{c}
n-1 \\
j-1
\end{array}\right)\left(\frac{1}{2}\right)^{n-1} f(0)+\int_{0}^{\infty}\left[\frac{(n-1) !}{(j-1) !(n-j-1) !}(1-F(\epsilon))^{j-1} F(\epsilon)^{n-j-1}\right.\right. \\
& \left.\left.-\frac{(n-1) !}{(j-2) !(n-j) !}(1-F(\epsilon))^{j-2} F(\epsilon)^{n-j}\right] f^{2}(\epsilon) d \epsilon\right] .
\end{aligned}
$$

And when there is no threshold, $t_{j}-e=-\infty$ for all $j$, so the equilibrium effort $e$ is given by

$$
\begin{aligned}
c^{\prime}(e)= & \sum_{j=1}^{n} v\left(A_{j}\right)\left[\int _ { - \infty } ^ { \infty } \left[\frac{(n-1) !}{(j-1) !(n-j-1) !}(1-F(\epsilon))^{j-1} F(\epsilon)^{n-j-1}\right.\right. \\
& \left.\left.-\frac{(n-1) !}{(j-2) !(n-j) !}(1-F(\epsilon))^{j-2} F(\epsilon)^{n-j}\right] f^{2}(\epsilon) d \epsilon\right] .
\end{aligned}
$$

Thus, regardless of whether the mechanism designer uses the optimal threshold or no threshold, the equilibrium level of effort is pinned down by an equation of the form $c^{\prime}(e)=\sum_{j=1}^{n} b_{j} v\left(A_{j}\right)$ for some constants $b_{j}$.

Now, when $v(A)=A$, the equilibrium level of effort is pinned down by an equation of the form $c^{\prime}(e)=\sum_{j=1}^{n} b_{j} A_{j}$ for some constants $b_{j}$, so $c^{\prime}(e)$ and thus the equilibrium level of effort $e$ must be maximized by setting $A_{j}=A$ for one particular $j$ and $A_{j}=0$ for all other $j$. And since a winnertake-all prize structure elicits higher effort than awarding all of the prize to some other place, it then follows that the equilibrium level of effort must be maximized by using a winner-take-all prize structure regardless of whether the mechanism designer uses the optimal threshold or no threshold.

(2) As in part (1), regardless of whether the mechanism designer uses the optimal threshold or no threshold, the level of effort is pinned down by an equation of the form $c^{\prime}(e)=\sum_{j=1}^{n} b_{j} v\left(A_{j}\right)$ for some constants $b_{j}$. However, since the constants $b_{j}$ are different under the optimal threshold than under no threshold, in general the division of the prizes that maximizes $c^{\prime}(e)=\sum_{j=1}^{n} b_{j} v\left(A_{j}\right)$ 
subject to the constraint that $\sum_{j=1}^{n} A_{j}=A$ will be different under the optimal threshold than under no threshold for strictly concave $v(\cdot)$.

In particular, suppose there are at most two prizes, let $b_{j, o p t}$ denote the value of the constant $b_{j}$ under the optimal threshold, and let $b_{j, 0}$ denote the value of $b_{j}$ under no threshold. We know from Equations (19) and (20) that

$$
\begin{aligned}
b_{j, o p t}-b_{j, 0}= & {\left[\left(\begin{array}{c}
n-1 \\
j-1
\end{array}\right)\left(\frac{1}{2}\right)^{n-1} f(0)-\int_{-\infty}^{0}\left[\frac{(n-1) !}{(j-1) !(n-j-1) !}(1-F(\epsilon))^{j-1} F(\epsilon)^{n-j-1}\right.\right.} \\
& \left.\left.-\frac{(n-1) !}{(j-2) !(n-j) !}(1-F(\epsilon))^{j-2} F(\epsilon)^{n-j}\right] f^{2}(\epsilon) d \epsilon\right] \\
= & \int_{-\infty}^{0}\left[\frac{(n-1) !}{(j-1) !(n-j-1) !}(1-F(\epsilon))^{j-1} F(\epsilon)^{n-j-1}\right. \\
& \left.-\frac{(n-1) !}{(j-2) !(n-j) !}(1-F(\epsilon))^{j-2} F(\epsilon)^{n-j}\right] f(\epsilon)(f(0)-f(\epsilon)) d \epsilon
\end{aligned}
$$

Thus, $\quad b_{1, o p t}-b_{1,0}=\int_{-\infty}^{0}(n-1) F(\epsilon)^{n-2} f(\epsilon)(f(0)-f(\epsilon)) d \epsilon \quad$ and $\quad b_{2, o p t}-b_{2,0}=\int_{-\infty}^{0}(n-$ 1) $F(\epsilon)^{n-3}[(n-2)(1-F(\epsilon))-F(\epsilon)] f(\epsilon)(f(0)-f(\epsilon)) d \epsilon$. Thus, the ratio of the terms in the integrands corresponding to $b_{2, o p t}-b_{2,0}$ and $b_{1, o p t}-b_{1,0}$ is $\frac{(n-2)(1-F(\epsilon))-F(\epsilon)}{F(\epsilon)}=(n-2) \frac{1-F(\epsilon)}{F(\epsilon)}-1>$ $n-2-1=n-3 \geq 1$ for $n \geq 4$, where the first inequality follows from the fact that $F(\epsilon)<\frac{1}{2}$ and $\frac{1-F(\epsilon)}{F(\epsilon)}>1$ when $\epsilon<0$. Thus, $b_{2, o p t}-b_{2,0}>b_{1, o p t}-b_{1,0}$.

Now, we also know that $b_{1,0} \geq b_{2,0}$, because it is optimal to give more of the prize pool to the winner than to the second-place finisher. By combining this with the result in the previous paragraph, we know that $\frac{b_{2, o p t}}{b_{2,0}}>\frac{b_{1, \text { opt }}}{b_{1,0}}$. Thus, the division of the prizes $A_{j}$ that will maximize $c^{\prime}(e)=\sum_{j=1}^{2} b_{j} v\left(A_{j}\right)$ subject to the constraint that $A_{1}+A_{2}=A$ will give a larger value to the second prize under the optimal threshold than under no threshold.

\section{A.6 Endogenous Entry}

Proof of Theorem 7.1: We know from our results on exogenous entry in Theorem A.1 that when exactly $k$ agents participate in a threshold mechanism with rank-order rewards $\left(A_{1}, A_{2}, \ldots, A_{n}\right)$ and threshold $t$, there is a unique pure-strategy equilibrium in which all these $k$ agents exert the same level of effort $e^{*}(t, k)$ (under appropriate conditions on $c(\cdot)$ ). Since each agent observes the number of other participants prior to choosing her effort level, we will assume henceforth that if $k$ agents participate (for any $0 \leq k \leq n$ ), each of these agents chooses this equilibrium level of effort $e^{*}(t, k)$.

Now, let $k^{*}=k^{*}(t)$ denote the largest non-negative integer less than or equal to $n$ such that if exactly $k^{*}$ agents participate with effort $e^{*}\left(t, k^{*}\right)$, then no participating agent can profitably deviate by not participating. Some such $k^{*}$ exists, because if $k^{*}=0$, then this condition is vacuously satisfied. In this case, it is an equilibrium for exactly $k^{*}$ agents to participate, for the following reason: When $k^{*}$ agents participate, then no participant can profitably deviate by not participating in the contest, by the definition of $k^{*}$. Also, no non-participating agent can profitably deviate by participating in the contest-if an additional agent participated in the contest, then there would be exactly $k^{*}+1$ agents participating, and each of these agents would choose effort $e^{*}\left(t, k^{*}+1\right)$. But by the definition of $k^{*}$, we would then know that none of these participating agents would prefer to participate (since all agents are symmetric), meaning it was never profitable for this nonparticipating agent to deviate in the first place. Thus, there exists an equilibrium in which exactly $k^{*}$ agents participate and all of these agents exert the same level of effort upon participating. 
Proof of Theorem 7.2: If setting the threshold to $t^{*}(k)$ would not create an incentive for one of the $k$ participating agents not to participate, then we know from our results on exogenous entry that such a threshold would be optimal. If not, then some participating agent has an incentive not to participate at threshold $t^{*}(k)$. But we have seen in the proof of Theorem 4.3 that for any fixed level of participation, equilibrium effort $e^{*}(t)$ is increasing in the threshold $t$ for all thresholds $t \leq t^{*}(k)$. From this it follows that if the principal wants to maximize equilibrium effort subject to the constraint that exactly $k$ agents participate, and there is no equilibrium in which $k$ agents participate at threshold $t^{*}(k)$, then the maximum equilibrium effort with $k$ participants occurs at the largest threshold less than $t^{*}(k)$ at which there is an equilibrium in which exactly $k$ agents participate.

Proof of Theorem 7.3: To prove this result, we first illustrate that for any fixed level of participation, $k$, the expected utility of the agents in equilibrium is decreasing in the principal's choice of threshold for values of the threshold below $t^{*}$, the threshold where equilibrium effort and the threshold are equal. To see this, recall from Equation (10) that the equilibrium level of effort $e$ for a given threshold mechanism with threshold $t$ and prizes $A_{1}, \ldots, A_{k}$ is given by the following equation:

$$
\sum_{j=1}^{k}\left(v\left(A_{j}\right)-v\left(A_{j+1}\right)\right)\left[G_{j}(t-e) f(t-e)+\int_{t-e}^{\infty} \frac{d G_{j}(\epsilon)}{d \epsilon} f(\epsilon) d \epsilon\right]=c^{\prime}(e),
$$

where $G_{j}(q)$ denotes the probability that no more than $j-1$ of the $n-1$ values of $\epsilon_{k}$ are greater than $q$. The derivative of the left-hand side of this equation with respect to $t-e$ is

$$
\sum_{j=1}^{k}\left(v\left(A_{j}\right)-v\left(A_{j+1}\right)\right) G_{j}(t-e) f^{\prime}(t-e),
$$

which is positive when $t-e<0$. Now note that when $t<t^{*}$ that both equilibrium effort $e^{*}(t)$ and $t-e^{*}(t)$ are increasing in $t$ for the following reason: We have already seen in Theorem 4.3 that when $t<t^{*}$ that we have both $e^{*}(t)>t$ and that $e^{*}(t)$ is increasing in $t$. Thus, to prove this claim, we only need show that $t-e^{*}(t)$ is increasing in $t$ when $t<t^{*}$.

To see this, consider two thresholds $t_{1}$ and $t_{2}$ satisfying $t_{1}<t_{2}<t^{*}$. Let $e^{*}(t)$ denote the equilibrium level of effort when the mechanism designer uses a threshold $t$, and suppose by means of contradiction that $t_{1}-e^{*}\left(t_{1}\right) \geq t_{2}-e^{*}\left(t_{2}\right)$. Since we have seen that the left-hand side of Equation (21) is increasing in $t-e$ when $t-e<0$, it follows that the left-hand side of Equation (21) is greater when $t=t_{1}$ and $e=e^{*}\left(t_{1}\right)$ than when $t=t_{2}$ and $e=e^{*}\left(t_{2}\right)$. But since the right-hand side of Equation (21) is increasing in $e$, we also know that the right-hand side of Equation (21) is lower when $e=e^{*}\left(t_{1}\right)$ than when $e=e^{*}\left(t_{2}\right)$. This implies that Equation (21) cannot be simultaneously satisfied at $t=t_{1}$ and $e=e^{*}\left(t_{1}\right)$ as well as at $t=t_{2}$ and $e=e^{*}\left(t_{2}\right)$. This contradicts our assumption that $e^{*}\left(t_{1}\right)$ and $e^{*}\left(t_{2}\right)$ are equilibrium levels of effort corresponding to the thresholds $t_{1}$ and $t_{2}$, and illustrates that $t_{1}-e^{*}\left(t_{1}\right) \geq t_{2}-e^{*}\left(t_{2}\right)$ cannot hold. Thus, $t-e^{*}(t)$ is increasing in $t$ when $t<t^{*}$.

But this implies that both the equilibrium level of effort is increasing in $t$ for $t \leq t^{*}$ and the probability that the agents will meet the threshold in equilibrium is decreasing in $t$ for $t \leq t^{*}$ (since this probability is decreasing in $\left.t-e^{*}(t)\right)$. Thus, the expected utility of the agents is decreasing in the principal's choice of threshold for thresholds $t \leq t^{*}$. Now, we know that if there exists an equilibrium in which exactly $k_{1}$ agents participate when the threshold is $t_{1}$, then these $k_{1}$ agents all obtain non-negative utility in equilibrium when the threshold is $t_{1}$. And from the previous result in this paragraph, it follows that if the threshold is some $t_{2} \leq t_{1}$, then all $k_{1}$ of these agents would obtain non-negative utility in equilibrium if exactly $k_{1}$ agents participated. Thus, if it is not an 
equilibrium for exactly $k_{1}$ agents to participate when the threshold is $t_{2}$, then it must be the case that some non-participating agent can profitably deviate by participating when exactly $k_{1}$ agents participate. If we then let $k_{2}>k_{1}$ denote the largest integer such that no non-participating agent can profitably deviate by participating when the threshold is $t_{2}$ and exactly $k_{2}$ agents participate, then it is an equilibrium for exactly $k_{2}$ agents to participate when the threshold is $t_{2}$. The result then follows.

\section{ACKNOWLEDGMENT}

This work is based on an earlier work, "Cardinal Contests." In Proceedings of the 24th International Conference on the World Wide Web (WWW'15), ACM. Retrieved from http://dx.doi.org/10.1145/ 2736277.2741652 .

\section{REFERENCES}

[1] Nikolay Archak. 2010. Money, glory, and cheap talk: Analyzing strategic behavior of contestants in simultaneous crowdsourcing contests on topcoder.com. In Proceedings of the 19th International Conference on the World Wide Web (WWW'10). 21-30.

[2] Nikolay Archak and Arun Sundarajan. 2009. Optimal design of crowdsourcing contests. In Proceedings of the 30th International Conference on Information Systems (ICIS'09). Article 200.

[3] Johannes Gerd Becker and Damian S. Damianov. 2006. On the existence of symmetric mixed-strategy equilibria. Econ. Lett. 90, 1 (2006), 84-87.

[4] Kevin J. Boudreau, Nicola Lacetera, and Karim R. Lakhani. 2011. Incentives and problem uncertainty in innovation contests: An empirical analysis. Manage. Sci. 57, 5 (2011), 843-863.

[5] Jeffrey Carpenter, Peter Hans Matthews, and John Schirm. 2010. Sorting and incentive effects of pay-for-performance: An experimental investigation. Amer. Econ. Rev. 100, 1 (2010), 504-517.

[6] Timothy N. Cason, William A. Masters, and Roman M. Sheremeta. 2010. Entry into winner-take-all and proportionalprize contests: An experimental study. F. Public Econ. 94, 9-10 (2010), 604-611.

[7] Ruggiero Cavallo and Shaili Jain. 2013. Winner-take-all crowdsourcing contests with stochastic prediction. In Proceedings of the 1st AAAI Conference on Human Computation and Crowdsourcing (HCOMP'13). 34-41.

[8] Shuchi Chawla, Jason D. Hartline, and Balasubramanian Sivan. 2012. Optimal crowdsourcing contests. In Proceedings of the 23rd ACM-SIAM Symposium of Discrete Algorithms (SODA'12). 856-868.

[9] Yeon-Koo Che and Ian Gale. 2003. Optimal design of research tournaments. Amer. Econ. Rev. 93, 3 (2003), 646-671.

[10] Subhasish M. Chowdhury, Roman M. Sheremeta, and Theodore L. Turocyd. 2014. Overbidding and overspreading in rent-seeking experiments: Cost structure and prize allocation rules. Games Econ. Behav. 87 (2014), 224-238.

[11] Chen Cohen, Todd R. Kaplan, and Aner Sela. 2008. Optimal rewards in contests. RAND J. Econ. 39, 2 (2008), $434-451$.

[12] Dominic DiPalantino and Milan Vocnović. 2009. Crowdsourcing and all-pay contests. In Proceedings of the 10th ACM Conference on Electronic Commerce (EC'09). 119-128.

[13] Benjamin Edelman and Michael Schwarz. 2010. Optimal auction design and equilibrium selection in sponsored search auctions. Amer. Econ. Rev. Papers Proc. 100, 2 (2010), 597-602.

[14] Gerald Eisenkopf. 2014. The impact of management incentives in intergroup contests. Eur. Econ. Rev. 67 (2014), 42-61.

[15] Francesco Falluchi, Elke Renner, and Martin Sefton. 2013. Information feedback and contest structure in rent-seeking games. Eur. Econ. Rev. 64 (2013), 223-240.

[16] Qiang Fu and Jingfeng Lu. 2012. The optimal multi-stage contest. Econ. Theory 51, 2 (2012), 351-382.

[17] Qiang Fu, Jingfeng Lu, and Yue Pan. 2015. Team contests with multiple pairwise battles. Amer. Econ. Rev. 105, 7 (2015), $2120-2140$.

[18] Arpita Ghosh and R. Preston McAfee. 2012. Crowdsourcing with endogenous entry. In Proceedings of the 20th International World Wide Web Conference (WWW'12). 137-146.

[19] Amihai Glazer and Refael Hassin. 1988. Optimal contests. Econ. Inquiry 26, 1 (1988), 133-143.

[20] Jerry R. Green and Nancy L. Stokey. 1983. A comparison of tournaments and contracts. f. Politic. Econ. 91, 3 (1983), 349-364.

[21] Luisa Herbst, Kai A. Konrad, and Florian Morath. 2015. Endogenous group formation in experimental contests. Eur. Econ. Rev. 74 (2015), 163-189.

[22] Bengt Holmstrom. 1982. Moral hazard in teams. Bell f. Econ. 13, 2 (1982), 324-340.

[23] Todd R. Kaplan and David Wettstein. 2015. The optimal design of rewards in contests. Rev. Econ. Design 19, 4 (2015), 327-339.

[24] Kai A. Konrad. 2010. Dynamic Contests. Typescript. Max Planck Institute for Intellectual Property, Berlin. 
[25] Vijay Krishna and John Morgan. 1998. The winner-take-all principle in small tournaments. Adv. Appl. Microecon. 7 (1998), 61-74.

[26] Edward P. Lazear. 1989. Pay equality and industrial politics. F. Politic. Econ. 97, 3 (1989), 561-580.

[27] Edward P. Lazear and Sherwin Rosen. 1981. Rank-order tournaments as optimum labor contracts. F. Politic. Econ. 89, 5 (1981), 841-864.

[28] Bin Liu, Jingfeng Liu, Ruqu Wang, and Jun Zhang. 2013. Prize and Punishment: Optimal Contest Design with Incomplete Information. Typescript. National University of Signapore, Signapore.

[29] Tracy Xiao Liu, Jiang Yang, Lada A. Adamic, and Yan Chen. 2014. Crowdsourcing with all-pay auctions: A field experiment on taskcn. Manage. Sci. 60, 8 (2014), 2020-2037.

[30] Dylan B. Minor. 2013. Increasing Revenue through Rewarding the Best (or not at all). Typescript. Northwestern University, Evanston, IL.

[31] Benny Moldovanu and Aner Sela. 2001. The optimal allocation of prizes in contests. Amer. Econ. Rev. 91, 3 (2001), 542-558.

[32] Benny Moldovanu and Aner Sela. 2006. Contest architecture. F. Econ. Theory 126, 1 (2006), 70-96.

[33] Benny Moldovanu, Aner Sela, and Xianwen Shi. 2007. Contests for status. f. Politic. Econ. 115, 2 (2007), $338-363$.

[34] John Morgan, Henrik Orzen, and Martin Sefton. 2012. Endogenous entry in contests. Econ. Theory 51, 2 (2012), 435463.

[35] Barry J. Nalebuff and Joseph E. Stiglitz. 1983. Prizes and incentives: Towards a general theory of compensation and competition. Bell f. Econ. 14, 1 (1983), 21-43.

[36] Shmuel Nitzen and Kaoru Ueda. 2011. Prize sharing in collective contests. Eur. Econ. Rev. 55, 5 (2011), 678-697.

[37] Michael Ostrovsky and Michael Schwarz. 2016. Reserve Prices in Internet Advertising Auctions: A Field Experiment. Typescript. Stanford Graduate School of Business, Stanford, CA.

[38] Sérgio Parreiras and Anna Rubinchik. 2010. Contests with three or more heterogeneous agents. Games Econ. Behav. 68, 2 (2010), 703-715.

[39] James E. Praco, Amnon Rapoport, and Wilfred Amaldoss. 2005. Two-stage contests with budget constraints: An experimental study. J. Math. Psychol. 49, 4 (2005), 320-338.

[40] Curtis R. Price and Roman M. Sheremeta. 2011. Endowment effects in contests. Econ. Lett. 111, 3 (2011), $217-219$.

[41] Christian Riis. 2010. Efficient contests. J. Econ. Manage. Strategy 19, 3 (2010), 643-665.

[42] Roman M. Sheremeta. 2010. Experimental comparison of multi-stage and one-stage contests. Games Econ. Behav. 68, 2 (2010), 731-747.

[43] Ron Siegel. 2009. All-pay contests. Econometrica 77, 1 (2009), 71-92.

[44] Ron Siegel. 2010. Asymmetric contests with conditional investments. Amer. Econ. Rev. 100, 5 (2010), 2230-2260.

[45] Ron Siegel. 2012. Participation in deterministic contests. Econ. Lett. 116, 3 (2012), 588-592.

[46] Ron Siegel. 2014. Asymmetric contests with productive effort. Amer. Econ. 7.: Microecon. 6, 3 (2014), 59-105.

[47] Ron Siegel. 2014. Contests with productive effort. Int. 7. Game Theory 43, 3 (2014), 515-523.

[48] Stefan Szymanski and Tommaso M. Valletti. 2005. Incentive effects of second prizes. Eur. F. Politic. Econ. 21, 2 (2005), 467-481.

[49] Curtis R. Taylor. 1995. Digging for golden carrots: An analysis of research tournaments. Amer. Econ. Rev. 85, 4 (1995), 872-890.

[50] Jiang Yang, Lada A. Adamic, and Mark S. Ackerman. 2008. Crowdsourcing and knowledge sharing: Strategic user behavior on taskCN. In Proceedings of the 9th ACM Conference on Electronic Commerce (EC'08). 246-255.

[51] Husevin Yildirim. 2005. Contests with multiple rounds. Games Econ. Behav. 51, 1 (2005), 213-227.

Received September 2016; revised September 2017; accepted March 2018 